\title{
Revaluation of magnetic properties of Magneto, MagR and aGFP-TRPV1/GFP-ferritin
}

Guangfu Wang ${ }^{1,2}$, Peng Zhang ${ }^{1,2}$, Suresh K. Mendu ${ }^{1,2}$, Yali Wang ${ }^{1}$, Yajun Zhang ${ }^{1}$, Xi Kang ${ }^{1}$, Bimal N. Desai ${ }^{1,3}$, J. Julius Zhu ${ }^{1,3}$

${ }^{1}$ Department of Pharmacology, University of Virginia School of Medicine, Charlottesville, VA 22908, USA

Figures: 2; Supplemental Figures: 10

${ }^{2}$ These authors contributed equally to this work

${ }^{3}$ Correspondence and requests for materials should be addressed to Bimal Desai (bdesai@virginia.edu) (00000002-3928-5854) and Julius Zhu (iizhu@virginia.edu) (ORCID: 0000-0002-1879-983X)

Address for correspondence:

J. Julius Zhu

Department of Pharmacology

University of Virginia School of Medicine

1300 Jefferson Park Avenue

Charlottesville, VA 22908

Tel: (434) 243-9246

Fax: (434) 982-3878

Email: ïzhu@virginia.edu 


\section{ABSTRACT AND INTRODUCTION}

Magnetic control of neuronal activity offers many obvious advantages over electric, optogenetic and chemogenetic manipulations. A recent series of highly visible papers reported the development of magnetic actuators (i.e., Magneto, MagR and aGFP-TRPV1/GFP-ferritin) that appeared to be effective in controlling neuronal firing ${ }^{1-3}$, yet their action mechanisms seem to conflict with the principles of physics ${ }^{4}$. We found that neurons expressing Magneto, MagR and aGFP-TRPV1/GFP-ferritin did not respond to magnetic stimuli with any membrane depolarization (let alone action potential firing), although these neurons frequently generated spontaneous action potentials. Because the previous study did not establish the precise temporal correlation between magnetic stimuli and action potentials in recorded neurons ${ }^{1-3}$, the reported magnetically-evoked action potentials are likely to represent mismatched spontaneous firings.

\section{RESULTS}

To examine the membrane surface incorporation of Magneto, we transfected 293T cells with the P2A-linked wild type TRPV4 (the primogenitor of Magneto2.0), ferritin (the other key element of Magneto2.0) and mCherry, aka TRPV4-P2A-ferritin-P2A-mCherry, or the P2A-linked Magneto2.0 and mCherry, aka Magneto-P2A-mCherry. We then made simultaneous measurements of the magnetic stimulation- and agonist-evoked responses in control non-expressing and TRPV4-P2A-ferritin-P2A-mCherry or Magneto-P2A-mCherry expressing cell pairs (Fig 1a). To determine the precise timing of applied magnetic field, we used an LED illuminator and a photodetector to monitor the exact position of magnets mounted on a Luigs-Neumann JUNIOR COMPACT manipulator. Delivering a K\&J N42 neodymium 1/16" block magnet to the position 1,000 $\mu$ m away from recorded cells generated a 64.5-mT magnetic field (Fig S1). As expected, delivery and withdrawal of the magnet did not induce any current in control and TRPV4-P2A-ferritin-P2A-mCherry expressing cells (Fig 1b-c). In contrast, puff application of TRPV4 agonist, GSK1016790A (GSK101), reliably elicited inward currents in TRPV4-P2A-ferritinP2A-mCherry expressing cells, but not control non-expressing cells (Fig 1b-c). Additional analysis revealed that GSK101-elicited currents in TRPV4-P2A-ferritin-P2A-mCherry expressing cells had the IV relationship typical of TRPV4, and the currents were blocked by a TRPV4 antagonist GSK205 (Fig S2a-b), indicating TRPV4-specific currents $^{5}$. Surprisingly, neither the magnetic stimuli nor GSK101 induced any significant current in control and 
Magneto-P2A-mCherry expressing cells (Fig 1b-c). Together, these results suggest that unlike wild type TRPV4, the ferritin-conjugated variant of TRPV4, i.e., Magneto2.0, fails to form a functional ion channel and/or incorporate into the plasma membrane of 293T cells.

To test whether Magneto might evoke action potentials in neuronal cells, we first expressed mCherry-fused Magneto2.0, aka Magneto-Ts-mCherry, and Magneto-P2A-mCherry in CA1 neurons of cultured rat hippocampal slices using the established Sindbis viral expression system (Fig 1d; see $e^{6,7}$ for the methods). Two-photon images showed that Magneto-Ts-mCherry, although robustly expressed, seemed to have limited, if any, presence at the plasma membrane of CA1 neurons (Fig 1d insets). In consistent, Western blots showed that in contrast to TPRV4 in TRPV4-P2A-ferritin-P2A-mCherry expressing CA1 cells, Magneto2.0 membrane surface expression was minimal despite its high intracellular expression in Magneto-P2A-mCherry expressing CA1 cells (Fig 1e-g). To verify the results, we expressed Magneto-P2A-mCherry and TRPV4-P2A-ferritin-P2A-mCherry in the mouse barrel cortex in vivo for 7-10-days using the established lentiviral expression system (Fig S3; see $^{6}$ for the methods). Again, in spite of high intracellular expressions, only TPRV4, but not Magneto2.0, showed efficient membrane surface expression in barrel cortical neurons (Fig S3). These results are consistent with the fact that C-terminus of the Magneto2.0 primogenitor TRPV4, which is essential for surface trafficking and functional expression of TRPV4 ${ }^{5}$, is deleted in Magneto2. $0^{2}$. We then made simultaneous whole-cell recordings from pairs of control non-expressing and Magneto-P2A-mCherry expressing CA1 cells. Patch-clamp recordings showed that application of up to $64.5 \mathrm{mT}$ static magnetic field induced neither depolarization nor action potential firing in control and Magneto-P2A-mCherry expressing CA1 cells (Fig 1h-i). Similarly, the magnetic stimuli failed to induce depolarization and action potential discharge in Magneto-P2A-mCherry expressing CA1 cells when the expression was made with lentivirus (Fig S4). These results indicate that the magnetic stimuli do not induce action potential in Magneto expressing CA1 neurons in cultured slices.

To further examine Magneto, we made in vivo Sindbis viral expression of Magneto-P2A-mCherry in layer 2/3 pyramidal and stellate neurons in the mouse medial entorhinal cortex (MEC) or layer 5 pyramidal neurons in the mouse barrel cortex for $\sim 18 \mathrm{hrs}$, and then acutely prepared entorhinal or barrel cortical slices (Figs S5a and 
S6a). Simultaneous recordings showed that the $64.5 \mathrm{mT}$ static magnetic field did not induce any depolarization or action potential firing in control and Magneto-P2A-mCherry expressing neurons (Figs S4 and S6). We repeated the experiments using the same 3/8" permanent block magnet employed in the previous study ${ }^{2}$ (Fig S7a). Positioning the magnet $5.00 \mathrm{~mm}$ away from recorded neurons, which yielded a $78.8 \mathrm{mT}$ static magnetic field (Fig S1), induced neither depolarization nor action potential firing in control and Magneto-P2A-mCherry expressing entorhinal neurons (Fig S7b-c). In these experiments, we noted that control and Magneto-P2AmCherry expressing entorhinal neurons frequently displayed spontaneous synaptic events, and at times, the spontaneous events reached the threshold and triggered bursts of action potentials (Figs S5b, S5d and S7b), suggest a potential cause for the reported magnetic effects ${ }^{2}$.

We then recorded hippocampal neurons after $\sim 3-5$-week in vivo AAV viral expression of DIO-Magneto and GFPCre (Fig S8a), using the same brain slice tissues prepared and published in Wheeler et al. ${ }^{2}$. The 78.8 mT static magnetic field induced neither depolarization nor action potential discharge in control or Magneto expressing dentate gyrus neurons (Fig S8b-c). Finally, we made in vivo AAV viral expression of DIO-Magneto and GFPCre in layer 2/3 pyramidal and stellate neurons of mouse MEC for $\sim 3-5$ weeks, and then recorded activity of entorhinal neurons in acutely prepared entorhinal cortical slices (Fig 1j). Once again, simultaneous recordings showed that application of up to $64.5 \mathrm{mT}$ static magnetic field did not induce any depolarization or action potential firing in control and DIO-Magneto/GFP-Cre expressing entorhinal neurons (Fig 1k-I). Importantly, we observed abundant spontaneous activities that from time to time, reached the firing threshold and elicited action potentials in these experiments (Figs 1k, 1m, S8b and S8d). Collectively, our results consistently support the idea that Magneto does not function as an effective magnetic actuator and spontaneous action potentials can confound the interpretation of Magneto expressing neurons subjected to magnetic stimuli.

The above experiments raised the concern about the other two recently reported magnetic actuators, MagR and aGFP-TRPV1/GFP-ferritin, given no time correlation between magnetic stimuli and action potentials in MagR and aGFP-TRPV1/GFP-ferritin expressing neurons reported in the two other studies ${ }^{1,3}$. To test whether MagR may serve as a magnetic actuator, we made Sindbis viral expression of MagR-P2A-GFP in CA1 neurons of 
cultured rat hippocampal slices (Fig 2a). After 18-hour expression, we made simultaneous whole-cell recordings from pairs of control non-expressing and MagR-P2A-GFP expressing CA1 cells. Application of up to $64.5 \mathrm{mT}$ static magnetic field induced neither depolarization nor action potential firing in control and MagR-P2A-GFP expressing CA1 cells (Fig 2b-c). These results are indicative of no magnetic effect on MagR expressing CA1 neurons, and the previously reported MagR-mediated action potentials to be confounding spontaneous firing (cf. $\left.{ }^{8}\right)$.

We next examined whether aGFP-TRPV1/GFP-ferritin may serve as a magnetic actuator. In this experiment, we made Sindbis viral expression of aGFP-TRPV1-P2A-GFP-ferritinin hypothalamic neurons in intact mouse brains for 18 hours, and then made simultaneous whole-cell recordings from pairs of control non-expressing and aGFP-TRPV1/GFP-ferritin expressing hypothalamic neurons in acutely prepared slices (Fig 2 d). Following the previous report ${ }^{3}$, we delivered a K\&J N52 neodymium 1/16" cylinder magnet to the position $500 \mu$ m away from recorded cells, which generated a 108.0-mT magnetic field (Fig S1). Application of up to $108.0 \mathrm{mT}$ static magnetic induced neither depolarization nor action potential discharge in control and aGFP-TRPV1/GFP-ferritin expressing hypothalamic neurons (Fig 2e-f). Both control and aGFP-TRPV1/GFP-ferritin expressing hypothalamic neurons exhibited frequent spontaneous synaptic potentials, and at times, depolarizing spontaneous potentials reached the threshold to trigger a burst of action potentials (Figs $2 \mathbf{e}$ and $\mathbf{2 g}$ ). These results are suggestive of no magnetic effect on aGFP-TRPV1/GFP-ferritin expressing hypothalamic neurons and the previously reported aGFP-TRPV1/GFP-ferritin-mediated responses to be false positive magnetic effects caused by spontaneous activity.

\section{DISCUSSION}

In summary, we systematically investigated whether Magneto2.0 might function as a magnetic actuator with multiple approaches (i.e., transfection, Sindibis, lentivirus, and AAV viral expression in vitro and/or in vivo), multiple cell types (i.e., 293T cells, hippocampal CA1 neurons, dentate gyrus neurons, cortical L5 pyramidal neurons, entorhinal layer 2/3 stellate and pyramidal neurons), and multiple animal species (i.e., rats and mice). Our results, together with two accompanied studies that used additional approaches (e.g. viral expression), cell 
types (e.g., cerebral Purkinje neurons and barrel cortical layer 2/3 neurons), and manipulation/recording SFV methods (e.g., electric magnetic stimuli and in vivo recordings) ${ }^{9,10}$, consistently support the notion that Magneto2.0 does not serve as a magnetic actuator. Our experiments raise serious concerns of about the experimental design and execution of Wheeler et al. study. For example, deleting C-terminus of the Magneto2.0 primogenitor TRPV4 ${ }^{2}$, that is essential for surface trafficking of Magneto2. $0^{5}$, is poorly conceived. Moreover, whether the ferritin subunit-fused TRPV4s may multiplex together to form functional ferritins remains to be tested. Notably, Wheeler et al. used the recorded action potentials as the primary evidence to argue that Magneto2.0 serves as a magnetic actuator (and its surface expression) ${ }^{2}$, yet these electrophysiology recordings were poorly controlled and confounded by spontaneous action potentials. Thus, the rigorous electrophysiology experiment that is essential for validating Magneto as a magnetic actuator remains missing (also see ${ }^{11}$ with data suggestive of the endoplasmic reticulum as the source of minimal intracellular $\mathrm{Ca}^{2+}$ elevation). Indeed, with the location of stimulating magnets continuously monitored, multiple independent electrophysiological experiments in this study (some using the exact same magnetic stimulations and/or tissues prepared for Wheeler et al.), together with those in the two accompanied studies ${ }^{9,10}$, consistently demonstrated that Magneto did not respond to magnetic stimuli with any membrane depolarization (let alone action potential firing). We also show here that MagR and aGFP-TRPV1/GFP-ferritin do not produce any detectable magnetically-elicited membrane depolarization. Together, these results support the theoretical conclusion that Magneto, MagR and aGFP-TRPV1/GFP-ferritin are incapable of controlling neuronal activity by producing magnetically-evoked action potentials. We hope that our comprehensive testing of Magneto establishes a sample set of criteria to aid continuing tool-engineering efforts, including building of a magnetogenetic toolbox. The criteria include: first, surface expression validation; second, functional validation; and third, electrophysiological validation. Obviously, beyond the proof-of-principle, the applicability of novel tools is best ensured by using them to answer fundamental biology questions in a definitive manner, as was typical of previous developments in patch-clamp and imaging technology. 
ACKNOWLEDGEMENTS: We thank members of the Zhu laboratory for comments and technical assistance, many colleagues around the world for their encouragement, discussion, suggestions and/or sharing unpublished Magneto data. We also thank Dr. Can Xie for supplying clMagR, Drs. Sarah Stanley and Jeffrey Friedman for supplying aGFP-TRPV1/GFP-ferritin, and Drs Chris Deppmann, Ali Güler and/or Manoj Patel for supplying TRPV4-P2A-ferritin-P2A-mCherry, Magneto-P2A-mCherry and Magneto-Ts-mCherry constructs and AAV DIOMagneto and GFP-Cre viruses, and for the opportunity to visit and observe their electrophysiology experiments and multiple data cross-examination meetings in late 2015 after our many futile attempts to reproduce their findings. This study is supported in part by the Epilepsy Foundation postdoctoral fellowship No. 310443 (G.W.), Alzheimer's Association research fellowship (P.Z.), NIH grants GM108989 (B.N.D.) and NS104670 (J.J.Z.). J.J.Z. is the Radboud Professor and Sir Yue-Kong Pao Chair Professor.

\section{AUTHOR CONTRIBUTIONS}

G.W., P.Z. and S.K.M. carried out the experiments with assistance from Y.W, Y.Z. and X.K.; B.N.D. and J.J.Z. wrote the manuscript with input from all coauthors.

\section{DECLARATION OF INTERESTS}

The authors declare no competing interests. 


\section{METHODS}

\section{Animal preparation}

Male and female Sprague Dawley rats and C57BL/6 mice were used to prepare cultured slices and acute slices used in this study. Animals were maintained in the animal facility at the University of Virginia and family or pair housed in the temperature-controlled animal room with 12-h/12-h light/dark cycle. Food and water were available ad libitum. All procedures for animal surgery and maintenance were performed following protocols approved by the Animal Care \& Use Committee of the University of Virginia and in accordance with US National Institutes of Health guidelines.

\section{Cultured slice preparation}

Cultured slices were prepared from postnatal 6-7 day old rats or mice (P6-7) as reported in our previous studies $^{6,7}$. In brief, the hippocampi were dissected out in ice-cold HEPES-buffered Hanks' solution (pH 7.35) under sterile conditions, sectioned into 400- $\mathrm{mm}$ slices on a tissue chopper, and explanted onto a Millicell-CM membrane (0.4- $\mu \mathrm{m}$ pore size; Millipore). The membranes were then placed in $750 \mu \mathrm{l}$ of MEM culture medium, contained (in mM): HEPES 30, heat-inactivated horse serum 20\%, glutamine 1.4, D-glucose $16.25, \mathrm{NaHCO}_{3} 5$, $\mathrm{CaCl}_{2} 1, \mathrm{MgSO}_{4} 2$, insulin $1 \mathrm{mg} / \mathrm{ml}$, ascorbic acid $0.012 \%$ at $\mathrm{pH} 7.28$ and osmolarity 320 . Cultured slices were maintained at $35^{\circ} \mathrm{C}$, in a humidified incubator (ambient air enriched with $5 \% \mathrm{CO}_{2}$ ).

\section{Constructs of recombinant proteins and expression}

All constructs, including TRPV4-P2A-ferritin-P2A-mCherry, Magneto-P2A-mCherry and Magneto-Ts-mCherry were generously supplied by Drs Chris Deppmann and Ali Güler. Magneto-P2A-mCherry and Magneto-TsmCherry were subcloned into Sindbis and lenitiviral vectors. AAV viral solutions of the Cre-dependent Magneto2.0 AAV virus, aka AAV1-CMV::DIO-Magneto, and AAV9-Camk2a::EGFP-Cre were also supplied by Drs Chris Deppmann and Ali Güler. A P2A sequence was used to link cIMagR ${ }^{12}$ and GFP, and anti-GFP-TRPV1 and GFP-ferritin ${ }^{13}$ to create cIMagR-P2A-GFP and aGFP-TRPV1-P2A-GFP-ferritin, which were then subcloned into Sindbis viral vector. Construct expression followed our previous studies ${ }^{14,15}$. For expression in cultured 293T cells, Magneto-P2A-mCherry and TRPV4-P2A-ferritin-P2A-mCherry were transfected using the calcium 
phosphate transfection method. For expression in cultured slices, CA1 pyramidal neurons in hippocampal cultured slices were infected after 8-18 days in vitro with lentivirus or Sindbis virus, and then incubated on culture media and $5 \% \mathrm{CO}_{2}$ before experiments. For expression in intact brains, P18-28 mice were initially anesthetized by an intraperitoneal injection of ketamine and xylazine (10 and $2 \mathrm{mg} / \mathrm{kg}$, respectively). Animals were then placed in a stereotaxic frame and one or multiple small $(\sim 1 \times 1 \mathrm{~mm})$ holes were opened above the cortex. A glass pipette was used to make pressure injections of $\sim 100 \mathrm{nl}$ Sindbis or lentiviral solution, or $200 \mathrm{nl}$ equivolume mixture of $A A V$ viral solutions of AAV1-CMV::DIO-Magneto and AAV9-Camk2a::EGFP-Cre into the barrel cortex, hippocampus and/or MEC according to their stereotaxic coordinates. After injection, animals were allowed to recover from the anesthesia and returned to their cages. Experiments were typically performed within $18 \pm 2$ hours after Sindbis viral infection, 7-10 days after lentiviral infection and 3-5 weeks after AAV viral infection.

\section{Biochemical analysis}

Hippocampal extracts were prepared by homogenizing hippocampal CA1 regions isolated from cultured slices, while cortical extracts were prepared by homogenizing mCherry expressing barrel cortical areas isolated from acute cortical slices. Membranes were blotted with anti-FLAG antibody (1:5,000 for in vitro expression, 1:2,000 for in vivo expression; Fisher Scientific, Hampton, NH; Cat\# MA1-91878, RRID:AB_1957945), stripped and reblotted twice with anti-GluA1 (1:1,000 for in vitro expression; 1:1,000 for in vivo expression; EMD Millipore, Burlington, MA; Cat\# AB1504, RRID:AB_2113602) or anti-GluA2 antibody (1:6,000 for in vitro expression; 1:6,000 for in vivo expression; EMD Millipore; Cat\# AB1768, RRID:AB_2313802). Western blots were quantified by chemiluminescence and densitometric scanning of the films under linear exposure conditions.

\section{Electrophysiology and two-photon imaging}

Simultaneous multiple whole-cell recordings were obtained from nearby expressing and non-expressing 293T cells, CA1 pyramidal neurons, barrel cortical layer 5 pyramidal neurons, dentate gyrus neurons, entorhinal layer $2 / 3$ stellate and pyramidal neurons, under visual guidance with fluorescence and transmitted light illumination ${ }^{7,16}$, using up to two Axopatch-200B (voltage clamp) or Axoclamp 2B (current clamp) amplifiers (Molecular Devices, LLC, Sunnyvale, CA). Bath solution $\left(29 \pm 1.5^{\circ} \mathrm{C}\right)$, unless otherwise stated, contained (in $\mathrm{mM}$ ): $\mathrm{NaCl} 119, \mathrm{KCl} 2.5$, 
$\mathrm{CaCl}_{2} 2, \mathrm{MgCl}_{2} 1, \mathrm{NaHCO}_{3} 26, \mathrm{NaH}_{2} \mathrm{PO}_{4} 1$, glucose 25, at $\mathrm{pH} 7.4$ and gassed with $5 \% \mathrm{CO}_{2} / 95 \% \mathrm{O}_{2}$. $\mathrm{Patch}$ recording pipettes (3-6 $\mathrm{M} \Omega$ ) for current (voltage-clamp) recordings contained (in $\mathrm{mM}$ ): cesium methanesulfonate 115, $\mathrm{CsCl}$ 20, HEPES 10, $\mathrm{MgCl}_{2}$ 2.5, $\mathrm{Na}_{2} \mathrm{ATP} 4, \mathrm{Na}_{3} \mathrm{GTP}$ 0.4, sodium phosphocreatine 10, EGTA 0.6, and spermine 0.1 , at $\mathrm{pH}$ 7.25; and for voltage (current-clamp) recordings contained: potassium gluconate 115, HEPES 10, $\mathrm{MgCl}_{2}$ 2, MgATP 2, $\mathrm{Na}_{2} \mathrm{ATP} 2, \mathrm{Na}_{3} \mathrm{GTP} 0.3$ and $\mathrm{KCl}$ 20, at $\mathrm{pH}$ 7.25.

Two-photon imaging and electrophysiology were simultaneously performed using a custom-built microscope operated by a custom-written IGOR Pro 6 program (WaveMetrics, Lake Oswego, OR) ${ }^{6,16}$. Neighboring expressing and non-expressing CA1 or dentate gyrus neuron pairs were broken in simultaneously to load green indicator Alexa $488(20 \mu \mathrm{M})$. Images were taken 15-30 minutes after loading of the indicator. Alexa 488 and mCherry were excited by a femtosecond Ti:Sapphire laser (Chameleon Ultra; Coherent, Santa Clara, CA) at a wavelength of $880 \mathrm{~nm}$.

\section{Agonist application and magnetic stimulation}

TRPV4 agonist, GSK1016790A (EMD Millipore, Billerica, MA), was puff applied with a brief (1 sec) air pressure by a glass pipette mounted on a Luigs-Neumann JUNIOR COMPACT manipulator (Luigs-Neumann GmbH, Ratingen, Germany) and positioned $\sim 150 \mu \mathrm{m}$ away from the recorded 293T cells. TRPV4-specific antagonist GSK205 (EMD Millipore), was bath applied. Magnetic stimuli were made using axially magnetized magnets, including a 3/8" cylinder magnet used in the previous study ${ }^{2}$ and N42 1/16" x 1/4" block neodymium magnets purchased from K\&J Magnetics. The magnetic intensity-distance relationships of these magnets were calculated with an HT20 Gauss Tesla meter (Shanghai Hengtong Cidian Technology Co., Ltd., Shanghai, China) (Fig S1). A Luigs-Neumann JUNIOR COMPACT manipulator was used to mount and rapidly position the magnets to 1.00 mm (for N42 block magnets), $0.50 \mathrm{~mm}$ (for N52 cylinder magnets) or $5.00 \mathrm{~mm}$ (for the 3/8" cylinder magnet) away from recorded cells to create a static magnetic field $>50 \mathrm{mT}$ or $>100 \mathrm{mT}$. After stimulation, the magnets were rapidly withdrawn by $12-15 \mathrm{~mm}$ to eliminate magnetic stimuli. To determine the precise timing of applied magnetic field, we used an LED illuminator and a photodetector (Thorlabs Inc, Newton, NJ) to monitor the exact position of magnets mounted on a Luigs-Neumann JUNIOR COMPACT manipulator. 


\section{Statistical analysis}

Statistical results were reported as meanıs.e.m. Animals or cells were randomly assigned into control or experimental groups and investigators were blinded to experiment treatments. Given the negative correlation between the variation and square root of sample number, $n$, the group sample size was typically set to be $\sim 10-25$ to optimize the efficiency and power of statistical tests. Statistical significances of the means $(p<0.05$; two sides) were determined using Wilcoxon non-parametric tests for paired samples. The data that support the findings of this study are available from the corresponding authors upon request. 
bioRxiv preprint doi: https://doi.org/10.1101/737254; this version posted August 27, 2019. The copyright holder for this preprint (which was not certified by peer review) is the author/funder, who has granted bioRxiv a license to display the preprint in perpetuity. It is made available under aCC-BY-NC-ND 4.0 International license.

\section{Figure 1}

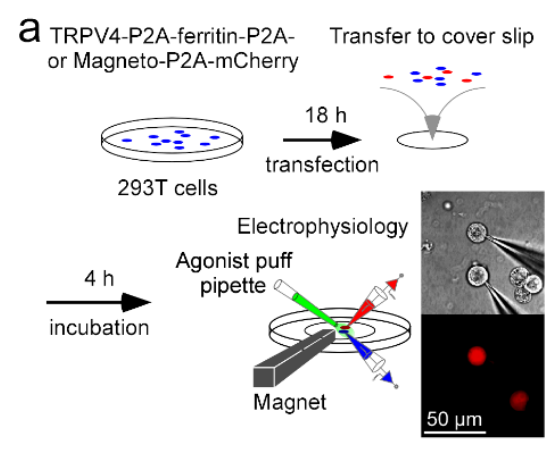

d

Magneto-Ts-mCherry
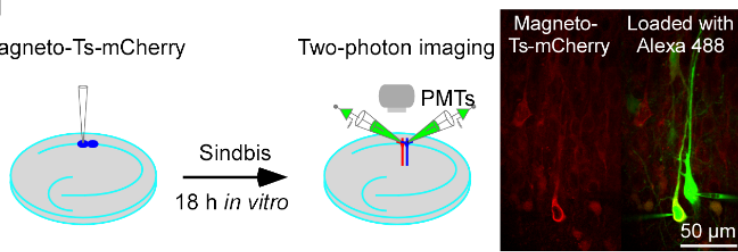

$\mathrm{f}$
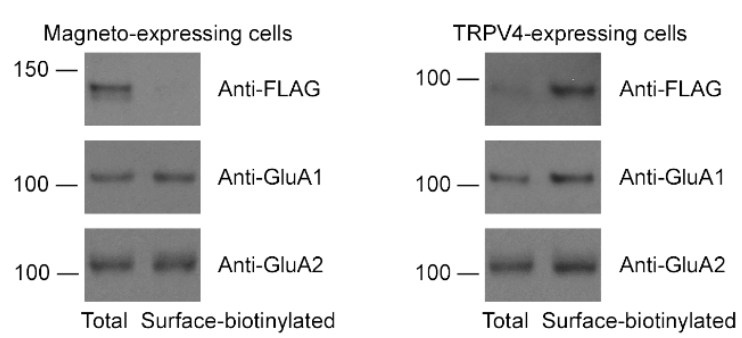

$\mathrm{h}$

- Control - Magneto - Magnet position

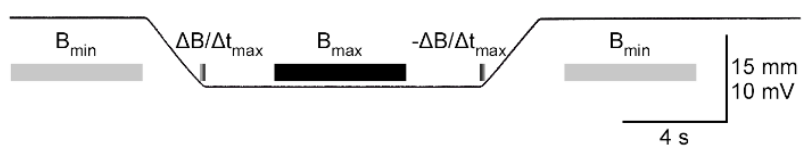

j

CMV::DIO-Magneto + Camk2a::Cre-EGFP

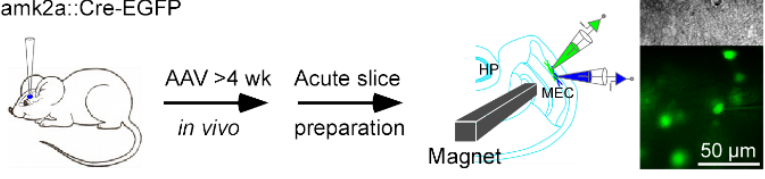

1

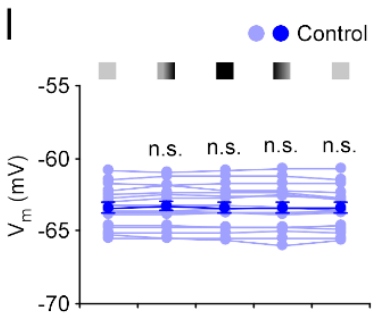

b $\quad-$ Control - TRPV4 - Magneto - Magnet position

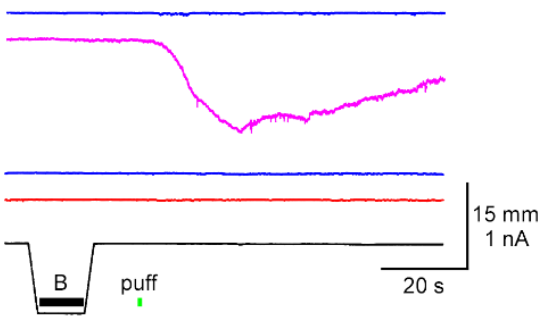

e

Magneto- or TRPV4- Western blotting Electrophysiology P2A-mCherry
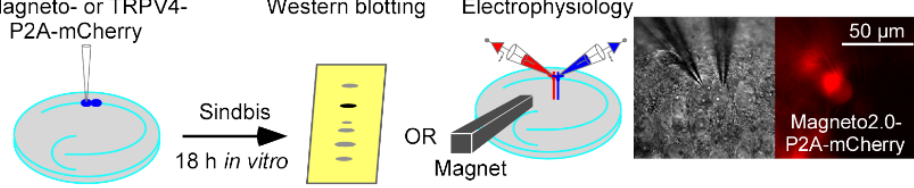

g
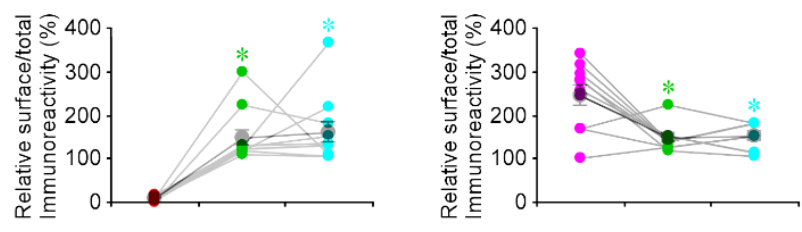

i
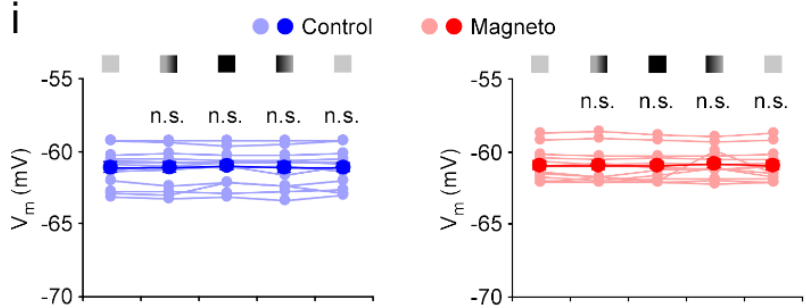

$\mathrm{k}$

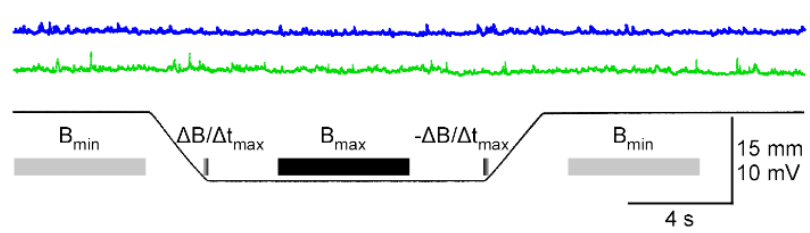

m

Spontaneous activity in control non-expressing $(-)$ and Magneto expressing $(-)$ neurons
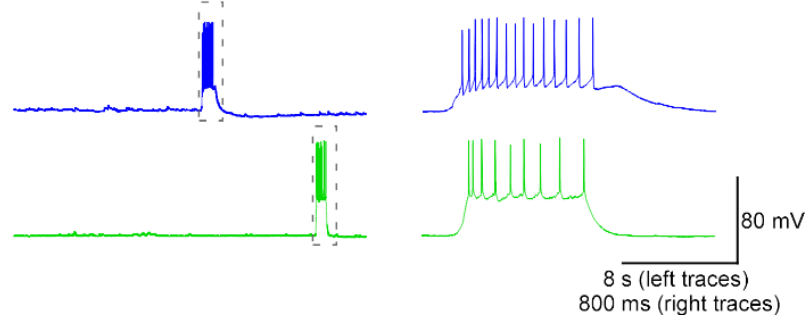


\section{Figure 1. No magnetic effect in cells or neurons expressing Magneto2.0.}

(a) Schematic drawing outlines the design of in vitro transfection, magnetic stimulation and electrophysiological recordings in TRPV4-P2A-ferritin-P2A-mCherry and Magneto-P2A-mCherry expressing cultured 293T cells. The right images show simultaneous whole-cell recordings from a pair of control non-expressing and MagnetoP2A-mCherry expressing cells under transmitted light (left) and fluorescence microscopy with RFP filter (right).

(b) Current recordings from neighboring control non-expressing and TRPV4-P2A-ferritin-P2A-mCherry expressing cells (left), and control non-expressing and Magneto-P2A-mCherry expressing cells (right) during magnetic stimulation and puff application of 100 nM TRPV4 agonist GSK1016790A (GSK101).

(c) Values of currents of control non-expressing and TRPV4-P2A-ferritin-P2A-mCherry expressing 293T cells during magnetic stimulation and puff application of GSK101 (Ctrl: $-0.5 \pm 0.9$ pA; EXP: $-1.8 \pm 1.1$ pA, $Z=-1.511$, $p=0.12$ for control cells; Ctrl: $9.0 \pm 5.3 p A$; EXP: $433.5 \pm 132.6 p A, Z=2.934, p<0.005$ for TRPV4 expressing cells; $n=11)$. Values of currents of control non-expressing and Magneto-P2A-mCherry expressing 293T cells during magnetic stimulation and puff application of GSK101 (Ctrl: $-0.2 \pm 0.6$ pA; EXP: $0.6 \pm 1.4 p A, Z=-0.175, p=0.86$ for control cells; Ctrl: 4.1 \pm 2.2 pA; EXP: $7.1 \pm 2.0$ pA, $Z=1.293, p=0.20$ for Magneto expressing cells; $n=13)$. Asterisk indicates $p<0.05$ (Wilcoxon tests).

(d) Schematic drawing outlines the design of in vitro Sindbis viral expression and two-photon imaging in cultured rat hippocampal slices. The right two-photon images show a pair of control non-expressing and Magneto-TsmCherry expressing CA1 pyramidal neurons after loading Alexa 488 with patch-clamp pipettes (left: mCherry channel only; right: mCherry and Alexa 488 red channels overlay).

(e) Schematic drawing outlines the design of in vitro Sindbis viral expression, biochemistry analysis, magnetic stimulation and electrophysiological recordings in cultured rat hippocampal slices. The right images show simultaneous whole-cell recordings from a pair of control non-expressing and Magneto-P2A-mCherry expressing CA1 pyramidal neurons under transmitted light (left) and fluorescence microscopy with RFP filter (right).

(f) Western blots of total and membrane surface-biotinylated recombinant Magneto2.0 and TRPV4 (both of which are FLAG tagged), and endogenous GluA1 and GluA2 in CA1 cells prepared from cultured rat hippocampal slices. Each lane loaded with $20 \mu \mathrm{g}$ proteins. 


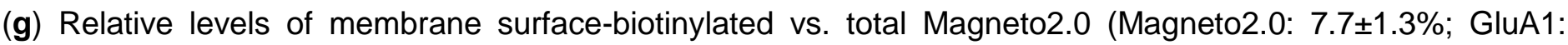
148.0 $\pm 17.8 \%, n=11, Z=2.934, p<0.005$; GluA2: 160.2 $\pm 23.3 \% ; n=11, Z=2.934, p<0.005$ ) and TRPV4 (TRPV4: 245.4 $\pm 24.0 \%$; GluA1: $145.5 \pm 9.4 \%, n=10, Z=-2.396, p<0.05 ;$ GluA2: $152.7 \pm 10.1 \%, n=10, Z=-2.396, p<0.05)$ compared to GluA1 and GluA2. Asterisks indicate $p<0.05$ (Wilcoxon tests).

(h) Recordings of membrane potentials of the pair of control non-expressing and Magneto-P2A-mCherry expressing CA1 pyramidal neurons before, during and after magnetic stimuli delivered with a K\&J N42 1/16" permanent block magnet mounted on a micromanipulator.

(i) Values of membrane potentials of control non-expressing (Initial $B_{\min }:-61.1 \pm 0.3 \mathrm{mV} ; \Delta \mathrm{B} / \Delta \mathrm{t}_{\max }:-61.2 \pm 0.4 \mathrm{mV}$, $Z=-1.038, p=0.28 ; B_{\max }:-61.1 \pm 0.3 \mathrm{mV}, Z=-0.105, p=0.92 ;-\Delta \mathrm{B} / \Delta \mathrm{t}_{\max }:-61.2 \pm 0.4 \mathrm{mV}, Z=-0.364, p=0.70 ;$ Ending $B_{\min }:-61.1 \pm 0.3 \mathrm{mV}, Z=1.083, p=0.28$; Wilcoxon tests) and Magneto-P2A-mCherry (Initial $B_{\min }:-60.9 \pm 0.3 \mathrm{mV}$; $\Delta \mathrm{B} / \Delta \mathrm{t}_{\max }:-61.0 \pm 0.3 \mathrm{mV}, Z=-0.664, p=0.51 ; \mathrm{B}_{\max }:-61.0 \pm 0.3 \mathrm{mV}, Z=-1.103, p=0.31 ;-\Delta \mathrm{B} / \Delta \mathrm{t}_{\max }:-60.8 \pm 0.3 \mathrm{mV}, Z=-$ 0.314, $p=0.75$; Ending $B_{\min }:-61.0 \pm 0.3 \mathrm{mV}, Z=-0.734, p=0.46$; Wilcoxon tests) expressing CA1 pyramidal neurons when the permanent magnet was away from (light), approaching to (light-dark transient color), close to (dark), retracting from (dark-light transient color), and away from (light) recorded neurons ( $n=13$ from 6 animals). Note dark lines/dotes indicating averages and shaded lines/dotes indicating experimental data. Note also no difference in membrane potential in control non-expressing and Magneto-P2A-mCherry expressing CA1 pyramidal neurons in all the experimental stages ( $p>0.05$; Wilcoxon tests).

(j) Schematic drawing outlines the design of in vivo AAV viral expression of CMV::DIO-Magneto and Camk2a::Cre-EGFP, ex vivo magnetic stimulation and electrophysiological recordings in acutely prepared mouse MEC slices. The right images show simultaneous whole-cell recordings from a pair of control nonexpressing and DIO-Magneto/Cre-GFP expressing MEC layer 2/3 pyramidal neurons under transmitted light (left) and fluorescence microscopy with GFP middle filter (right).

(k) Recordings of membrane potentials of the pair of control non-expressing and DIO-Magneto/Cre-GFP expressing MEC layer 2/3 neurons before, during and after magnetic stimuli delivered with a K\&J N42 1/16" permanent block magnet mounted on a micromanipulator.

(I) Values of membrane potentials of control non-expressing (Initial $B_{\min }$ : $-63.4 \pm 0.3 \mathrm{mV} ; \Delta \mathrm{B} / \Delta \mathrm{t}_{\max }:-63.4 \pm 0.4 \mathrm{mV}$, $Z=1.018, p=0.31 ; B_{\max }:-63.4 \pm 0.4 \mathrm{mV}, Z=0.213, p=0.83 ;-\Delta \mathrm{B} / \Delta \mathrm{t}_{\max }:-63.4 \pm 0.4 \mathrm{mV}, Z=0.734, p=0.46$; Ending $B_{\min }$ : 
$-63.4 \pm 0.3 \mathrm{mV}, Z=-1.065, p=0.29)$ and Cre-GFP/DIO-Magneto2.0 (Initial $\mathrm{B}_{\min }:-63.4 \pm 0.3 \mathrm{mV} ; \Delta \mathrm{B} / \Delta \mathrm{t}_{\max }:-63.4 \pm 0.4$ $\mathrm{mV}, Z=-0.024, p=0.98 ; B_{\max }:-63.4 \pm 0.4 \mathrm{mV}, Z=0.166, p=0.87 ;-\Delta \mathrm{B} / \Delta \mathrm{t}_{\max }:-63.4 \pm 0.4 \mathrm{mV}, Z=0.166, p=0.87 ;$ Ending $B_{\min }:-63.4 \pm 0.3 \mathrm{mV}, Z=-1.207, p=0.23$ ) expressing MEC L2/3 pyramidal and stellate neurons when the permanent magnet was away from (light), approaching to (light-dark transient color), close to (dark), retracting from (darklight transient color), and away from (light) recorded neurons ( $n=17$ from 11 animals). Note dark lines/dotes indicating averages and shaded lines/dotes indicating experimental data. Note also no difference in membrane potential in control non-expressing and DIO-Magneto/Cre-GFP expressing MEC L2/3 neurons in all the experimental stages ( $p>0.05$; Wilcoxon tests).

(m) Recordings of spontaneous events in the pair of control non-expressing and DIO-Magneto/Cre-GFP expressing MEC layer 2/3 neurons. Note that the spontaneous suprathreshold events in the gray dash line boxes are shown again in an expanded time scale in the right. 


\section{Figure 2}

a

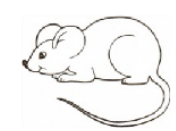

Cultured slice

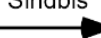

$18 \mathrm{~h}$ in vitro preparation

clMag-P2A-GFP

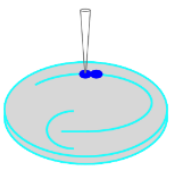

Electrophysiology
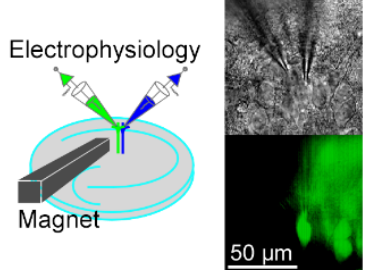

b - Control - MagR - Magnet position

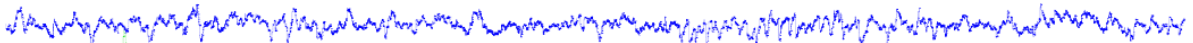

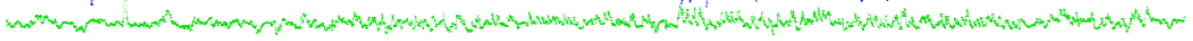

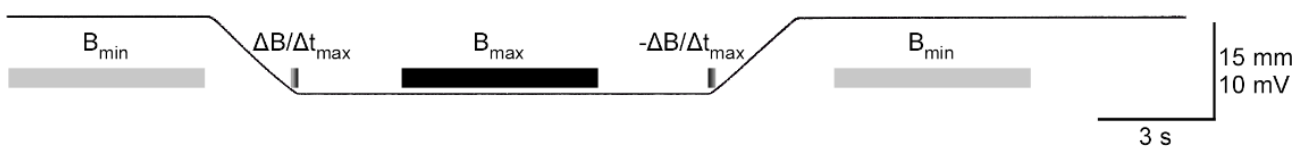

C
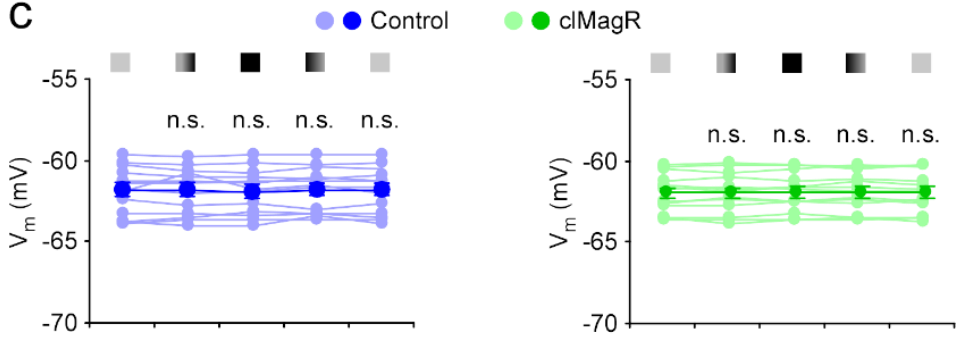

d

XGPP-TRPV1/GFP-Ferritin

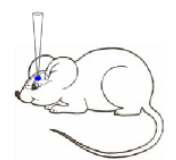

$\underbrace{\text { Sindbis }}_{18 \mathrm{~h} \text { in vivo }}$

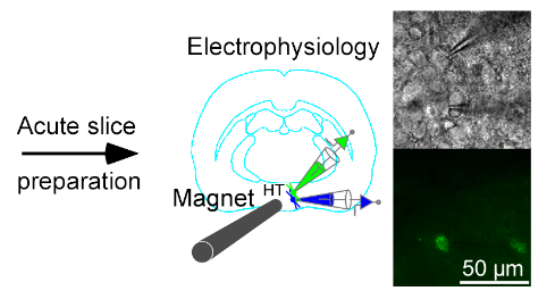

e - Control - aGFP-TRPV1/GFP-Ferritin - Magnet position

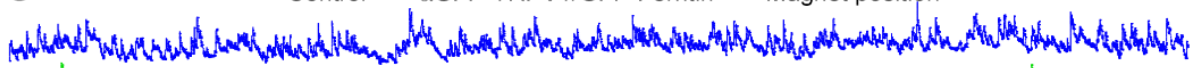

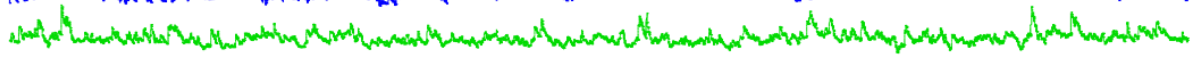

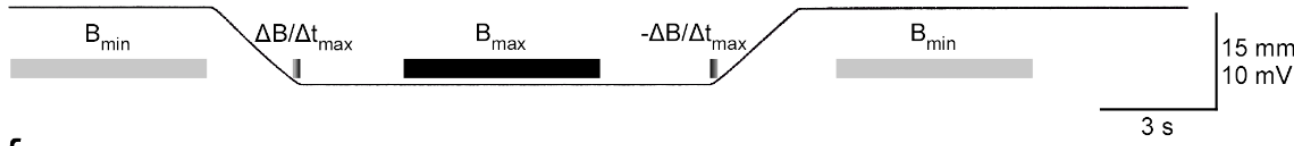

$f$

- Control $\alpha$ GFP-TRPV1/GFP-Ferritin

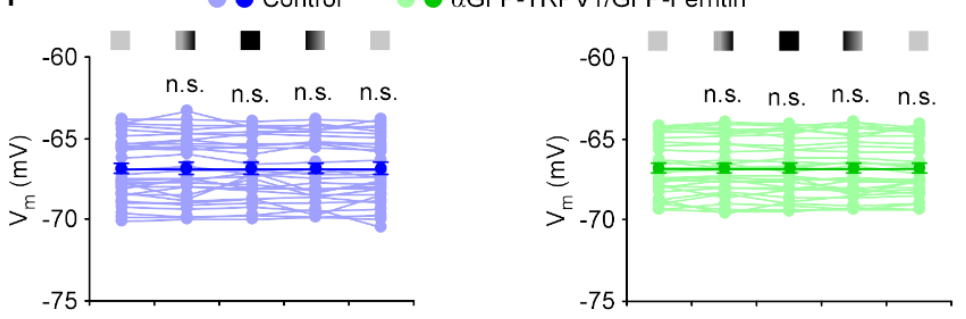

9 Spontaneous activity in control non-expressing (-) and Anti-GFP-TRPV1/GFP-Ferritin expressing (-) neurons
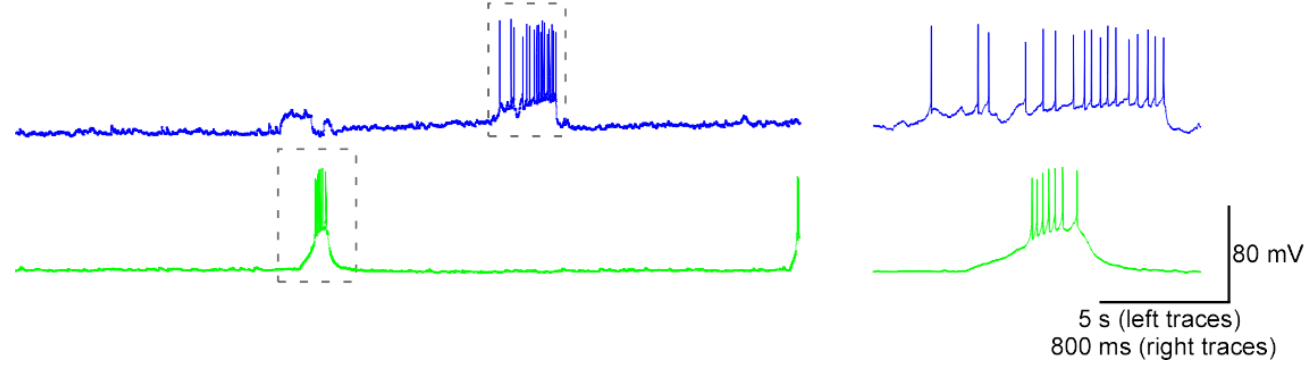

Figure 2. No magnetic effect in neurons expressing MagR or aGFP-TRPV1/GFP-ferritin.

(a) Schematic drawing outlines the design of in vitro Sindbis viral expression, magnetic stimulation and electrophysiological recordings in cultured rat hippocampal slices. The right images show simultaneous whole- 
cell recordings from a pair of control non-expressing and MagR expressing CA1 pyramidal neurons under transmitted light (left) and fluorescence microscopy with GFP middle filter (right).

(b) Recordings of membrane potentials of the pair of control non-expressing and MagR expressing CA1 pyramidal neurons before, during and after magnetic stimuli delivered with a K\&J N42 1/16" permanent block magnet mounted on a micromanipulator.

(c) Values of membrane potentials of control non-expressing (Initial $\mathrm{B}_{\min }$ : $-62.0 \pm 0.4 \mathrm{mV} ; \Delta \mathrm{B} / \Delta \mathrm{t}_{\max }:-62.0 \pm 0.4 \mathrm{mV}$, $Z=-0.934, p=0.35 ; B_{\max }:-62.0 \pm 0.4 \mathrm{mV}, Z=-0.804, p=0.42 ;-\Delta \mathrm{B} / \Delta \mathrm{t}_{\max }:-61.9 \pm 0.4 \mathrm{mV}, Z=0.804, p=0.42 ;$ Ending $B_{\min }$ : $-62.0 \pm 0.4 \mathrm{mV}, Z=0.035, p=0.97$; Wilcoxon tests) and MagR (Initial $\mathrm{B}_{\min }:-62.0 \pm 0.3 \mathrm{mV} ; \Delta \mathrm{B} / \Delta \mathrm{t}_{\max }:-62.0 \pm 0.3 \mathrm{mV}$, $Z=0.175, p=0.86 ; B_{\max }:-61.9 \pm 0.3 \mathrm{mV}, Z=1.433, p=0.15 ;-\Delta \mathrm{B} / \Delta \mathrm{t}_{\max }:-61.9 \pm 0.3 \mathrm{mV}, Z=1.013, p=0.31 ;$ Ending $\mathrm{B}_{\min }$ : $-62.0 \pm 0.3 \mathrm{mV}, Z=0.874, p=0.38$; Wilcoxon tests) expressing $\mathrm{CA} 1$ pyramidal neurons when the permanent magnet was away from (light), approaching to (light-dark transient color), close to (dark), retracting from (darklight transient color), and away from (light) recorded neurons ( $n=13$ from 6 animals). Note dark lines/dotes indicating averages and shaded lines/dotes indicating experimental data. Note also no difference in membrane potential in control non-expressing and MagR expressing CA1 pyramidal neurons in all the experimental stages ( $p>0.05$; Wilcoxon tests).

(d) Schematic drawing outlines the design of in vivo Sindbis viral expression, ex vivo magnetic stimulation and electrophysiological recordings in acutely prepared mouse hypothalamic slices. The right images show simultaneous whole-cell recordings from a pair of control non-expressing and aGFP-TRPV1/GFP-ferritin expressing hypothalamic neurons under transmitted light (left) and fluorescence microscopy with GFP middle filter (right).

(e) Recordings of membrane potentials of the pair of control non-expressing and aGFP-TRPV1/GFP-ferritin expressing hypothalamic neurons before, during and after magnetic stimuli delivered with a K\&J N52 1/16" permanent cylinder magnet mounted on a micromanipulator.

(f) Values of membrane potentials of control non-expressing (Initial $B_{\min }:-67.0 \pm 0.3 \mathrm{mV} ; \Delta \mathrm{B} / \Delta \mathrm{t}_{\max }:-66.9 \pm 0.3 \mathrm{mV}$, $Z=0.999, p=0.32 ; B_{\max }:-66.9 \pm 0.3 \mathrm{mV}, Z=0.020, p=0.98 ;-\Delta \mathrm{B} / \Delta \mathrm{t}_{\max }:-67.0 \pm 0.4 \mathrm{mV}, Z=0.078, p=0.94 ;$ Ending $\mathrm{B}_{\min }$ : $-67.0 \pm 0.3 \mathrm{mV}, Z=-0.549, p=0.58$ ) and $\alpha G F P-T R P V 1 / G F P-f e r r i t i n\left(I n i t i a l ~ B_{\min }:-66.9 \pm 0.3 \mathrm{mV} ; \Delta \mathrm{B} / \Delta \mathrm{t}_{\max }:-66.8 \pm 0.3\right.$ $\mathrm{mV}, Z=0.588, p=0.56 ; \mathrm{B}_{\max }:-66.9 \pm 0.3 \mathrm{mV}, Z=0.020, p=0.98 ;-\Delta \mathrm{B} / \Delta \mathrm{t}_{\max }:-66.9 \pm 0.3 \mathrm{mV}, Z=0.353, p=0.72 ;$ Ending 
$\left.B_{\min }:-66.9 \pm 0.3 \mathrm{mV}, Z=-0.882, p=0.38\right)$ expressing hypothalamic neurons when the permanent magnet was away from (light), approaching to (light-dark transient color), close to (dark), retracting from (dark-light transient color), and away from (light) recorded neurons ( $n=31$ from 13 animals). Note dark lines/dotes indicating averages and shaded lines/dotes indicating experimental data. Note also no difference in membrane potential in control nonexpressing and aGFP-TRPV1/GFP-ferritin expressing hypothalamic neurons in all the experimental stages ( $p>0.05$; Wilcoxon tests).

(g) Recordings of spontaneous events in the pair of control non-expressing and aGFP-TRPV1/GFP-ferritin expressing hypothalamic neurons. Note that the spontaneous suprathreshold events in the gray dash line boxes are shown again in an expanded time scale in the right. 


\section{Figure S1}

a

K\&J N52 neodymium $1 / 16 " \times 1 / 4 "$ cylinder magnet

K\&J N42 neodymium $1 / 16 " \times 1 / 4$ " block magnet

3/8" cylinder magent (from Drs Patel \& Güler) b

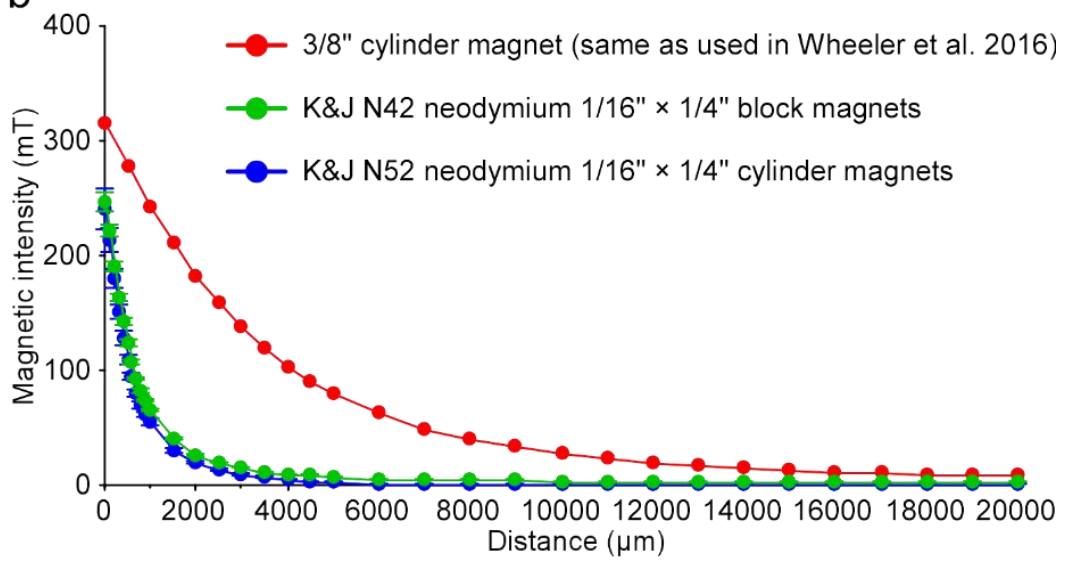

Figure S1. Properties of magnets used in experiments.

(a) Image shows the two frequently used magnets attached to a $2 \mathrm{~mm}$ diameter borosilicate glass or a $4 \mathrm{~mm}$ diameter stainless steel rod.

(b) Plots of the magnetic intensity against the distance of N42 neodymium 1/16" $\times 1 / 4$ " block magnets (K\&J Magentics, Inc.; $n=4$ ) and a 3/8" cylinder magnet (the same as used in the previous report ${ }^{2} ; n=1$ ). 


\section{Figure S2}

a

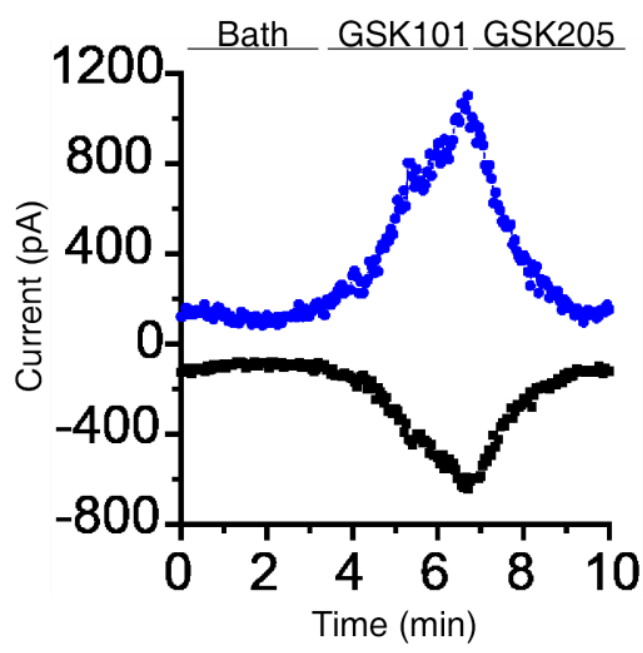

b

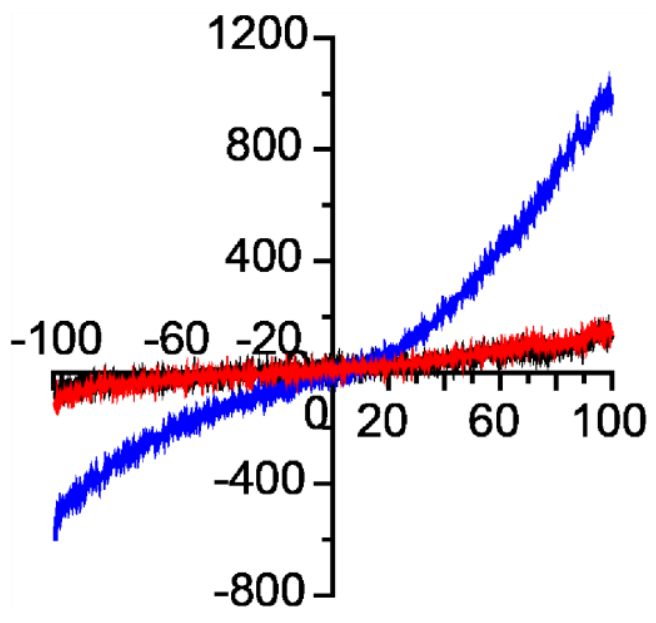

C

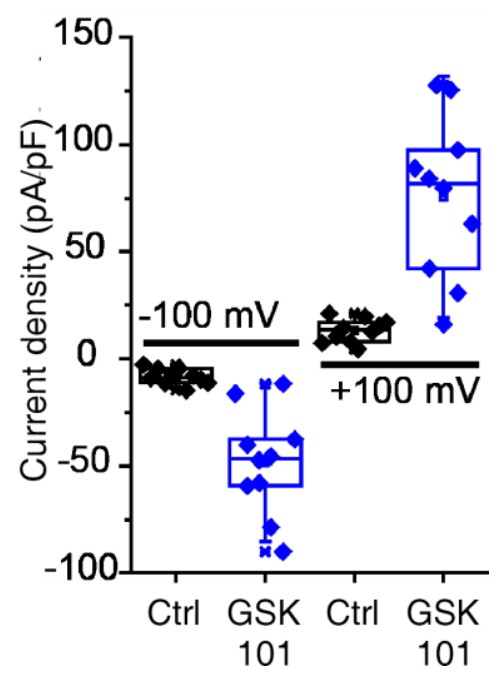

Figure S2. TRPV4-specific currents in TRPV4-P2A-ferritin-P2A-mCherry expressing 293T cells.

(a) Whole-cell patch-clamp recordings of outward (at $100 \mathrm{mV}$, blue trace) and inward (at -100mV, black trace) currents in 293T cells expressing control TRPV4-P2A-ferritin-P2A-mCherry in response to TRPV4 agonist GSK101 (10 nM) and antagonist GSK205 (10 $\mu \mathrm{M})$.

(b) Current-voltage relationships of control (black trace), the GSK101- (blue trace) and GSK205- (red trace) evoked responses in 293T cells expressing TRPV4-P2A-ferritin-P2A-mCherry.

(c) Box charts showing quantification of the peak currents in 293T cells expressing TRPV4-P2A-ferritin-P2AmCherry (Ctrl: $-8.3 \pm 1.2$ pA; GSK101: $-48.5 \pm 7.8$ pA; $n=10, Z=-2.803, p<0.01$, for inward currents at $-100 \mathrm{mV}$; Ctrl:

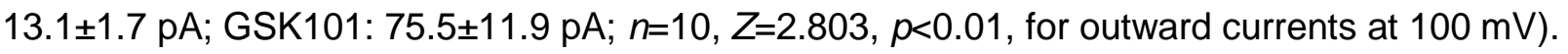




\section{Figure S3}

a

Magneto- or TRPV4P2A-mCherry

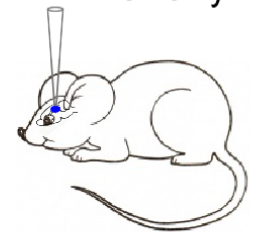

\section{Lentivirus Acute slice}

$7-10 \mathrm{~d}$ in vivo preparation
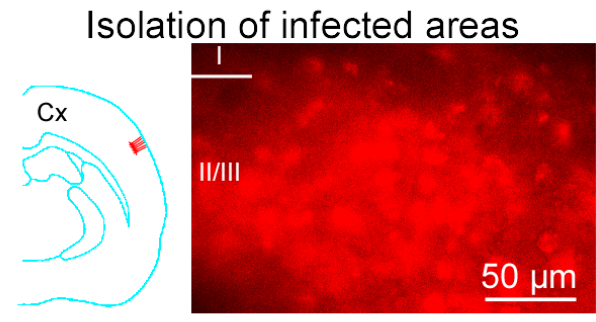

Western blotting

b

Magneto-expressing cells

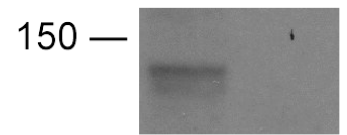

Anti-FLAG

TRPV4-expressing cells
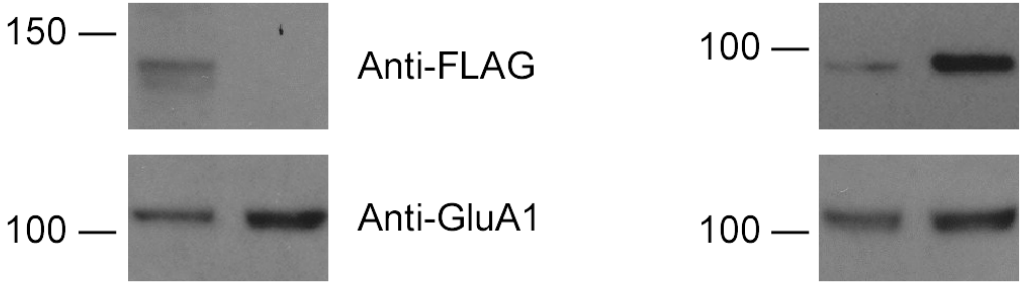

Anti-FLAG

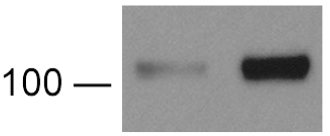

Anti-GluA2

Total Surface-

biotinylated
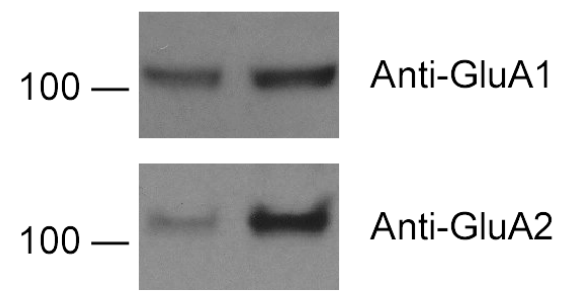

Total Surface-

biotinylated

C

- Magneto

TRPV4

- GluA1 - GluA2

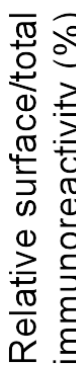
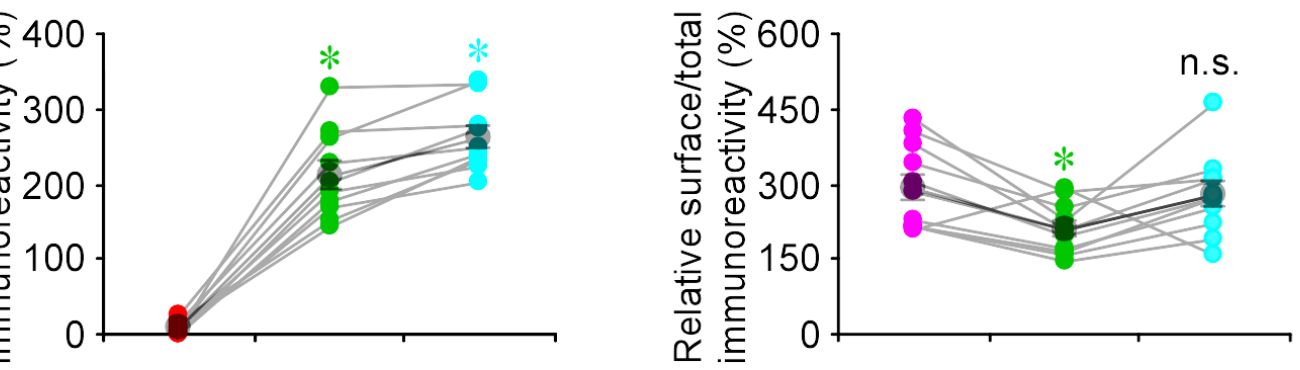

n.s.

Figure S3. Membrane surface trafficking of Magneto2.0 is impaired in barrel cortical cells in vivo.

(a) Schematic drawing outlines the design of biochemistry analysis of Magneto2.0 and TRPV4 (both of which are FLAG tagged) expressed in the intact mouse barrel cortex with lentivirus. The inset image shows the expressing barrel cortical area identifiable by mCherry fluorescence. Note the majority of barrel cortical neurons nearby the viral injected site expressing mCherry.

(b) Western blots of total and membrane surface-biotinylated recombinant Magneto2.0 and TRPV4, and endogenous GluA1 and GluA2 in barrel cortical neurons after 7-10 days of expression. Each lane loaded with $40 \mu \mathrm{g}$ proteins. 
bioRxiv preprint doi: https://doi.org/10.1101/737254; this version posted August 27, 2019. The copyright holder for this preprint (which was not certified by peer review) is the author/funder, who has granted bioRxiv a license to display the preprint in perpetuity. It is made available under aCC-BY-NC-ND 4.0 International license.

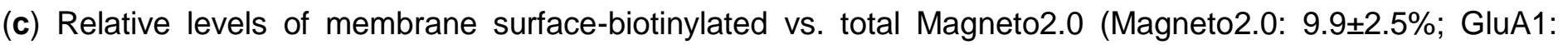
212.2 $\pm 18.7 \%, n=10, Z=2.803, p<0.01 ;$ GluA2: $260.3 \pm 14.2 \% ; n=12, Z=2.803, p<0.01$ ) and TRPV4 (TRPV4: 293.2 $\pm 25.4 \%$; GluA1: $209.9 \pm 14.6 \%, n=11, Z=-2.401, p<0.05 ;$ GluA2: $276.2 \pm 24.3 \%, n=11, Z=-1.607, p=0.29)$ compared to GluA1 and GluA2. Asterisks indicate $p<0.05$ (Wilcoxon tests). 


\section{Figure S4}

a
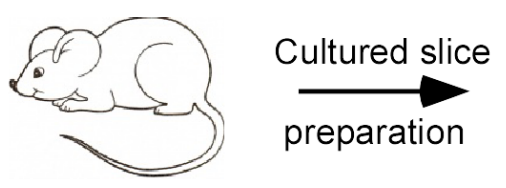

$$
\text { M }
$$

Magneto-P2A-mCherry

b

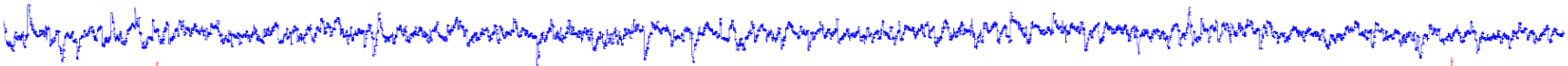

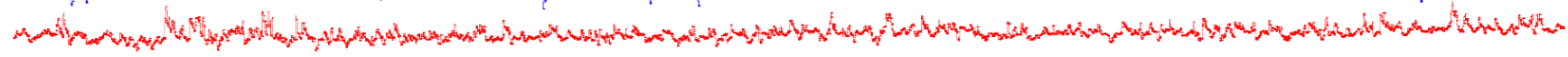
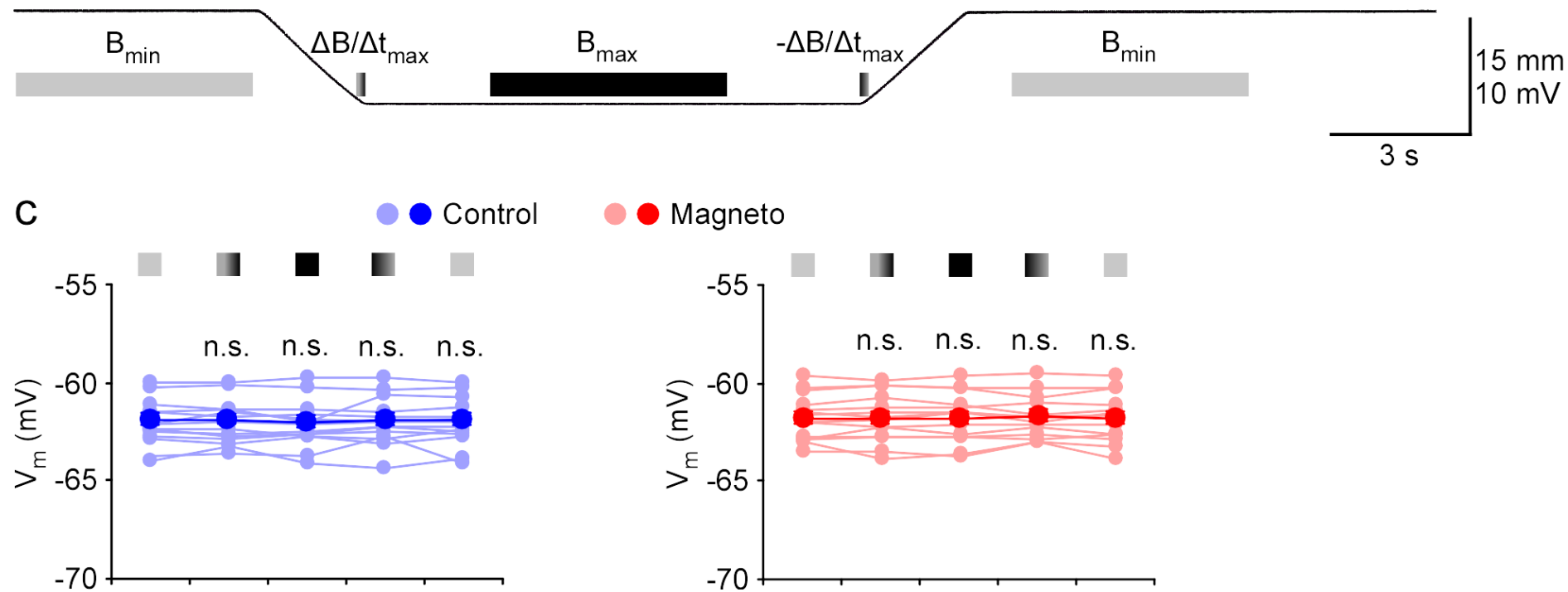
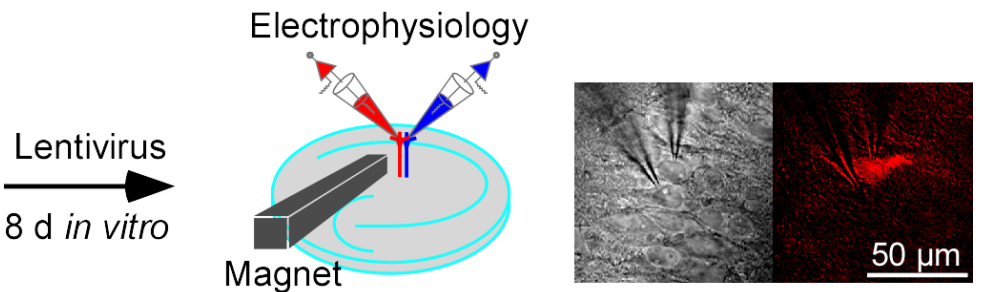

Figure S4. No magnetic effect in rat CA1 neurons expressing lentivirus-delivered Magneto2.0.

(a) Schematic drawing outlines the design of in vitro lentiviral expression, magnetic stimulation and electrophysiological recordings in cultured rat hippocampal slices. The right images show simultaneous wholecell recordings from a pair of control non-expressing and Magneto-P2A-mCherry expressing CA1 pyramidal neurons under transmitted light (left) and fluorescence microscopy with RFP filter (right).

(b) Recordings of membrane potentials of the pair of control non-expressing and Magneto-P2A-mCherry expressing CA1 pyramidal neurons before, during and after magnetic stimuli delivered with a K\&J N42 1/16" permanent block magnet mounted on a micromanipulator.

(c) Values of membrane potentials of control non-expressing (Initial $\mathrm{B}_{\min }:-62.0 \pm 0.3 \mathrm{mV} ; \Delta \mathrm{B} / \Delta \mathrm{t}_{\max }:-61.9 \pm 0.3 \mathrm{mV}$, $Z=-0.220, p=0.83 ; B_{\max }:-62.0 \pm 0.3 \mathrm{mV}, Z=-0.471, p=0.64 ;-\Delta \mathrm{B} / \Delta \mathrm{t}_{\max }:-61.9 \pm 0.3 \mathrm{mV}, Z=0.282, p=0.78$; Ending $B_{\min }$ : $-61.9 \pm 0.3 \mathrm{mV}, Z=1.287, p=0.20$; Wilcoxon tests) and Magneto-P2A-mCherry (Initial $\mathrm{B}_{\min }:-61.8 \pm 0.3 \mathrm{mV} ; \Delta \mathrm{B} / \Delta \mathrm{t}_{\max }$ : 
$-61.8 \pm 0.3 \mathrm{mV}, Z=0.282, p=0.78 ; \mathrm{B}_{\max }:-61.8 \pm 0.3 \mathrm{mV}, Z=0.659, p=0.51 ;-\Delta \mathrm{B} / \Delta \mathrm{t}_{\max }:-61.7 \pm 0.3 \mathrm{mV}, Z=-0.220$, $p=0.83$; Ending $B_{\min }:-61.8 \pm 0.3 \mathrm{mV}, Z=0.345, p=0.73$; Wilcoxon tests) expressing $\mathrm{CA} 1$ pyramidal neurons when the permanent magnet was away from (light), approaching to (light-dark transient color), close to (dark), retracting from (dark-light transient color), and away from (light) recorded neurons ( $n=14$ from 6 animals). Note dark lines/dotes indicating averages and shaded lines/dotes indicating experimental data. Note also no difference in membrane potential in control non-expressing and Magneto-P2A-mCherry expressing CA1 pyramidal neurons in all the experimental stages ( $p>0.05$; Wilcoxon tests). 


\section{Figure S5}

a Magneto-P2A-mCherry
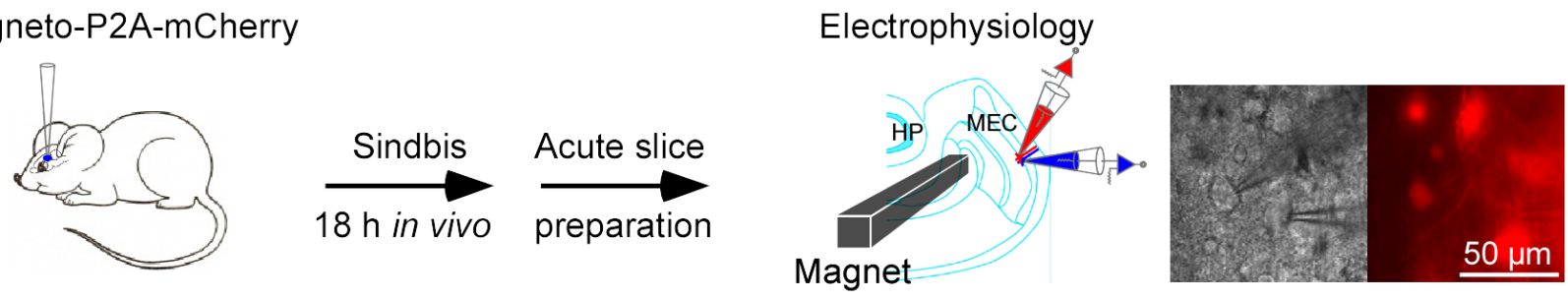

b - Control - Magneto - Magnet position

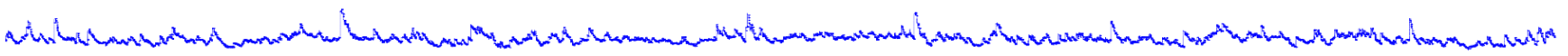

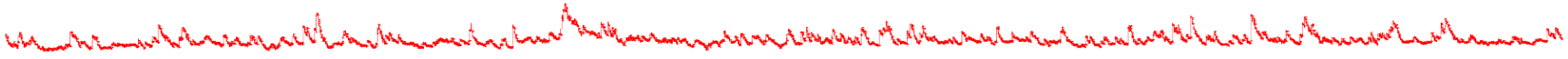

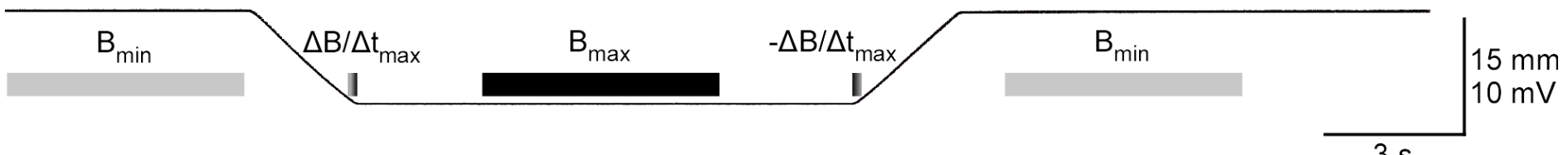

C

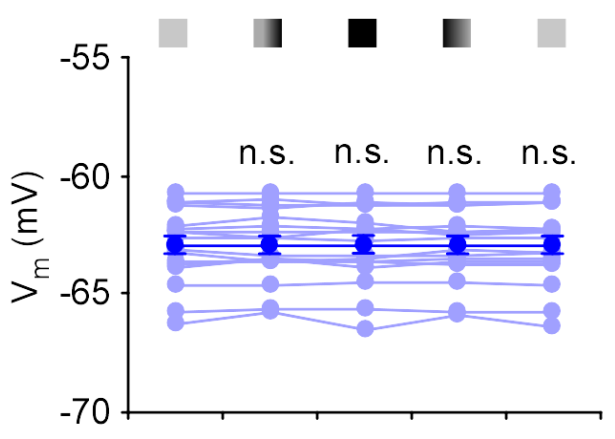

Magneto

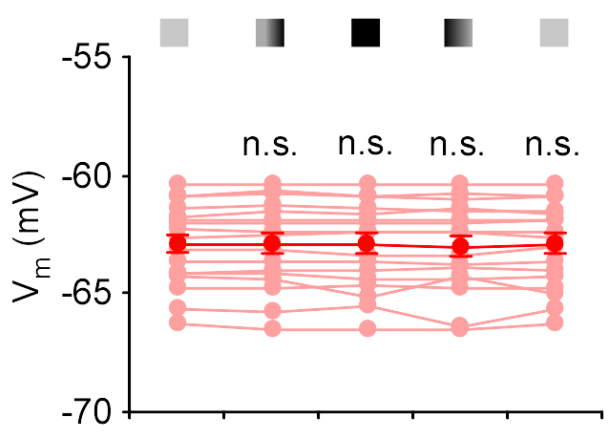

d Spontaneous activity in control non-expressing (-) and Magneto expressing (-) neurons
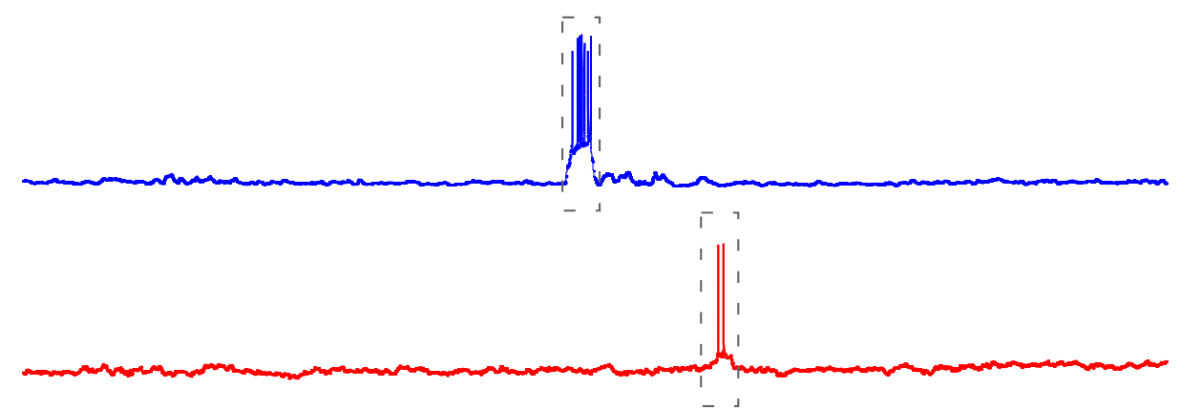
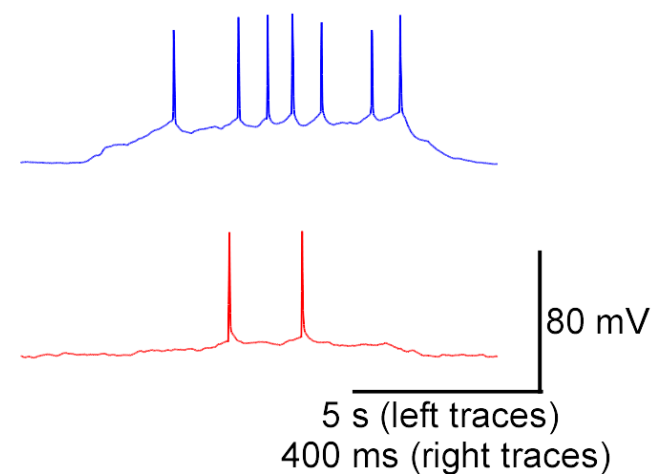

Figure S5. No magnetic effect in mouse MEC L2/3 neurons expressing Sindbis-delivered Magneto2.0.

(a) Schematic drawing outlines the design of in vivo Sindbis viral expression, ex vivo magnetic stimulation and electrophysiological recordings in acutely prepared mouse MEC slices. The right images show simultaneous 
whole-cell recordings from a pair of control non-expressing and Magneto-P2A-mCherry expressing MEC layer 2/3 pyramidal neurons under transmitted light (left) and fluorescence microscopy with RFP middle filter (right).

(b) Recordings of membrane potentials of the pair of control non-expressing and Magneto expressing MEC layer 2/3 neurons before, during and after magnetic stimuli delivered with a K\&J N42 1/16" permanent block magnet mounted on a micromanipulator.

(c) Values of membrane potentials of control non-expressing (Initial $\mathrm{B}_{\min }:-62.8 \pm 0.4 \mathrm{mV} ; \Delta \mathrm{B} / \Delta \mathrm{t}_{\max }:-62.8 \pm 0.4 \mathrm{mV}$, $Z=-0.024, p=0.98 ; B_{\max }:-62.8 \pm 0.4 \mathrm{mV}, Z=-0.828, p=0.41 ;-\Delta \mathrm{B} / \Delta \mathrm{t}_{\max }:-62.8 \pm 0.4 \mathrm{mV}, Z=-0.260, p=0.80 ;$ Ending $\mathrm{B}_{\min }:-62.8 \pm 0.4 \mathrm{mV}, Z=-0.923, p=0.36$ ) and Magneto-P2A-mCherry (Initial $\mathrm{B}_{\min }:-62.9 \pm 0.4 \mathrm{mV} ; \Delta \mathrm{B} / \Delta \mathrm{t}_{\max }:-62.9 \pm 0.4$ $\mathrm{mV}, Z=0.402, p=0.69 ; B_{\max }:-63.0 \pm 0.4 \mathrm{mV}, Z=-0.166, p=0.87 ;-\Delta \mathrm{B} / \Delta \mathrm{t}_{\max }:-63.0 \pm 0.5 \mathrm{mV}, Z=-1.065, p=0.29 ;$ Ending $B_{\min }$ : $63.0 \pm 0.4 \mathrm{mV}, Z=0.166, p=0.87$ ) expressing MEC L2/3 pyramidal and stellate neurons when the permanent magnet was away from (light), approaching to (light-dark transient color), close to (dark), retracting from (darklight transient color), and away from (light) recorded neurons ( $n=17$ from 13 animals). Note dark lines/dotes indicating averages and shaded lines/dotes indicating experimental data. Note also no difference in membrane potential in control non-expressing and Magneto-P2A-mCherry expressing MEC L2/3 neurons in all the experimental stages ( $p>0.05$; Wilcoxon tests).

(d) Recordings of spontaneous events in the pair of control non-expressing and Magneto-P2A-mCherry expressing MEC layer 2/3 neurons. Note that the spontaneous suprathreshold events in the gray dash line boxes are shown again in an expanded time scale in the right. 


\section{Figure S6}

a Magneto-P2A-mCherry
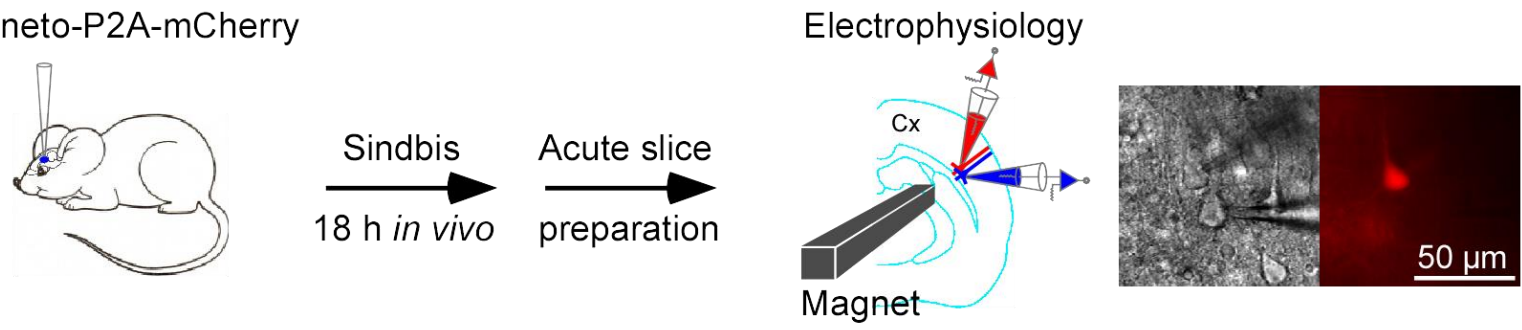

b

- Control - Magneto - Magnet position

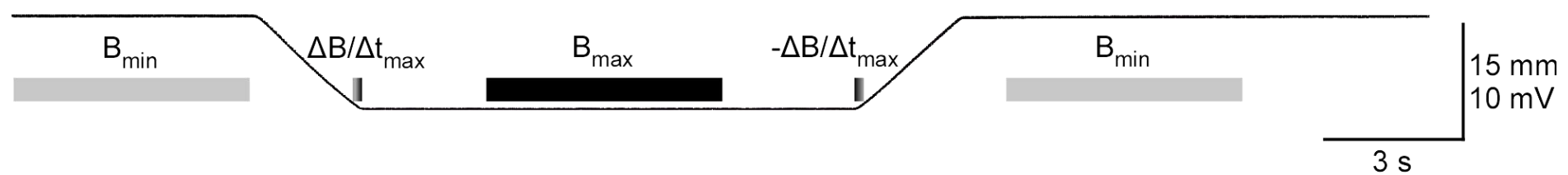

C

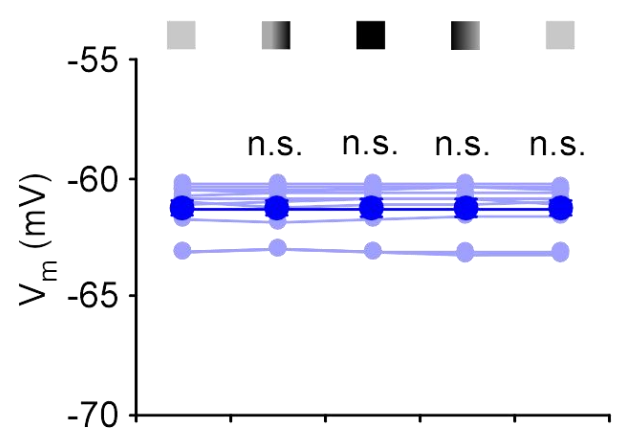

Magneto

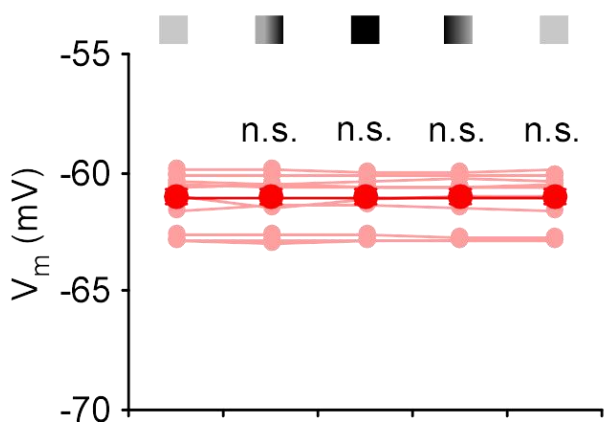

Figure S6. No magnetic effect in mouse barrel cortical L5 neurons expressing Magneto2.0.

(a) Schematic drawing outlines the design of in vivo Sindbis viral expression, ex vivo magnetic stimulation and electrophysiological recordings in acutely prepared mouse cortical slices. The right images show simultaneous whole-cell recordings from a pair of control non-expressing and Magneto-P2A-mCherry expressing barrel cortical L5 pyramidal neurons under transmitted light (left) and fluorescence microscopy with RFP middle filter (right). Cx: cerebral cortex.

(b) Recordings of membrane potentials of the pair of control non-expressing and Magneto-P2A-mCherry expressing barrel cortical L5 pyramidal neurons before, during and after magnetic stimuli delivered with a K\&J N42 1/16" permanent block magnet mounted on a micromanipulator. 
(c) Values of membrane potentials of control non-expressing (Initial $B_{\min }:-61.2 \pm 0.3 \mathrm{mV} ; \Delta \mathrm{B} / \Delta \mathrm{t}_{\max }:-61.2 \pm 0.3 \mathrm{mV}$, $Z=0.235, p=0.81 ; B_{\max }:-61.2 \pm 0.3 \mathrm{mV}, Z=0.000, p=1.00 ;-\Delta \mathrm{B} / \Delta \mathrm{t}_{\max }:-61.2 \pm 0.3 \mathrm{mV}, Z=0.628, p=0.53 ;$ Ending $B_{\min }$ : $-61.2 \pm 0.3 \mathrm{mV}, Z=0.392, p=0.70$; Wilcoxon tests) and Magneto-P2A-mCherry (Initial $\mathrm{B}_{\min }:-61.1 \pm 0.3 \mathrm{mV} ; \Delta \mathrm{B} / \Delta \mathrm{t}_{\max }$ : $-61.1 \pm 0.3 \mathrm{mV}, Z=0.000, p=1.00 ; B_{\max }:-61.1 \pm 0.3 \mathrm{mV}, Z=-0.785, p=0.43 ;-\Delta \mathrm{B} / \Delta \mathrm{t}_{\max }:-61.1 \pm 0.3 \mathrm{mV}, Z=-0.235$ $p=0.81$; Ending $B_{\min }:-61.1 \pm 0.3 \mathrm{mV}, Z=0.000, p=1.00$; Wilcoxon tests) expressing barrel cortical pyramidal neurons when the permanent magnet was away from (light), approaching to (light-dark transient color), close to (dark), retracting from (dark-light transient color), and away from (light) recorded neurons ( $n=12$ from 6 animals). Note dark lines/dotes indicating averages and shaded lines/dotes indicating experimental data. Note also no difference in membrane potential in control non-expressing and Magneto-P2A-mCherry expressing barrel cortical L5 pyramidal neurons in all the experimental stages ( $p \geq 0.05$; Wilcoxon tests). 


\section{Figure S7}

a Magneto-P2A-mCherry
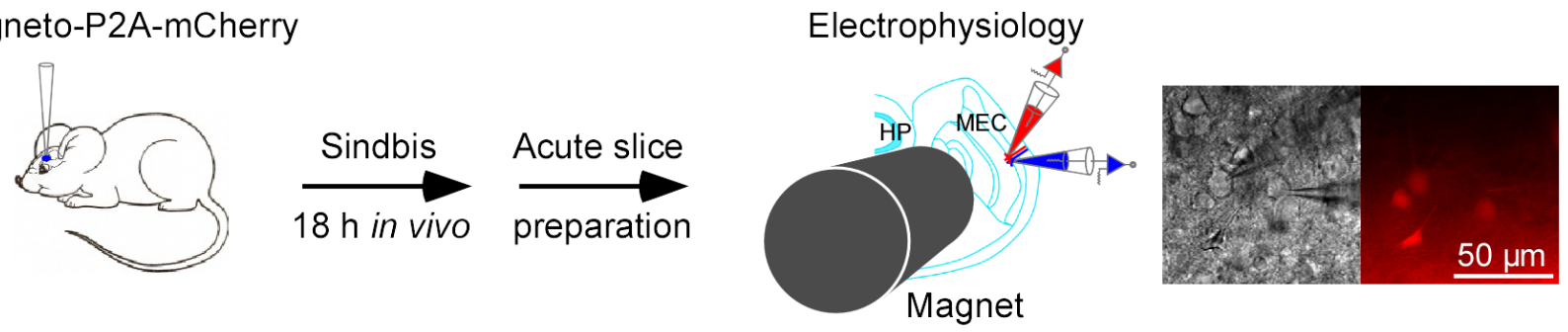

b - Control - Magneto - Magnet position

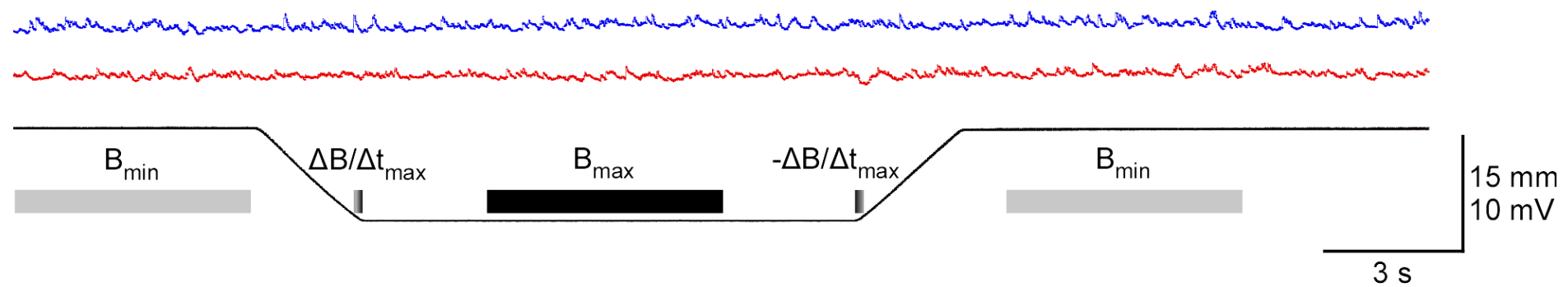

C

Control

Magneto
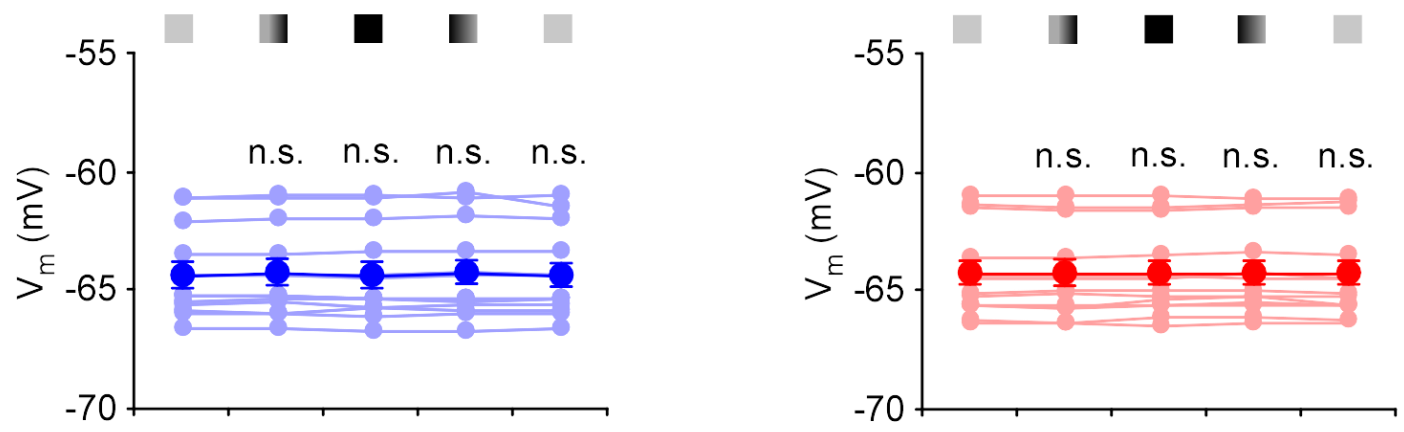

Figure S7. No 3/8" magnet-evoked effect in mouse entorhinal L2/3 neurons expressing Magneto2.0.

(a) Schematic drawing outlines the design of in vivo Sindbis viral expression, ex vivo magnetic stimulation and electrophysiological recordings in acutely prepared mouse MEC slices. The right images show simultaneous whole-cell recordings from a pair of control non-expressing and Magneto-P2A-mCherry expressing MEC L2/3 pyramidal neurons under transmitted light (left) and fluorescence microscopy with RFP middle filter (right). HP: hippocampus; MEC: medial entorhinal cortex.

(b) Recordings of membrane potentials of the pair of control non-expressing and Magneto-P2A-mCherry expressing MEC L2/3 pyramidal and stellate neurons before, during and after magnetic stimuli delivered with a 3/8" permanent block magnet mounted on a micromanipulator. 
(c) Values of membrane potentials of control non-expressing (Initial $\mathrm{B}_{\min }:-64.2 \pm 0.5 \mathrm{mV} ; \Delta \mathrm{B} / \Delta \mathrm{t}_{\max }:-64.2 \pm 0.5 \mathrm{mV}$, $Z=0.943, p=0.35 ; B_{\max }:-64.2 \pm 0.5 \mathrm{mV}, Z=-0.314, p=0.75 ;-\Delta \mathrm{B} / \Delta \mathrm{t}_{\max }:-64.2 \pm 0.5 \mathrm{mV}, Z=-1.363, p=0.17 ;$ Ending $B_{\min }:$ $-64.3 \pm 0.5 \mathrm{mV}, Z=-0.035, p=0.97 ;$ Wilcoxon tests) and Magneto-P2A-mCherry (Initial $B_{\min }$ : $-64.3 \pm 0.5 \mathrm{mV}$; $\Delta \mathrm{B} / \Delta \mathrm{t}_{\max }:-64.3 \pm 0.5 \mathrm{mV}, Z=-0.943, p=0.35 ; \mathrm{B}_{\max }:-64.3 \pm 0.5 \mathrm{mV}, Z=0.594, p=0.55 ;-\Delta \mathrm{B} / \Delta \mathrm{t}_{\max }:-64.3 \pm 0.5 \mathrm{mV}$ $Z=1.223, p=0.22$; Ending $B_{\min }:-64.3 \pm 0.5 \mathrm{mV}, Z=0.524, p=0.60$; Wilcoxon tests) expressing MEC L2/3 pyramidal and stellate neurons when the permanent magnet was away from (light), approaching to (light-dark transient color), close to (dark), retracting from (dark-light transient color), and away from (light) recorded neurons ( $n=13$ from 4 animals). Note dark lines/dotes indicating averages and shaded lines/dotes indicating experimental data. Note also no difference in membrane potential in control non-expressing and Magneto-P2A-mCherry expressing MEC L2/3 neurons in all the experimental stages ( $p>0.05$; Wilcoxon tests). 


\section{Figure S8}

a

CMV::DIO-Magneto

+ Camk2a::Cre-EGFP
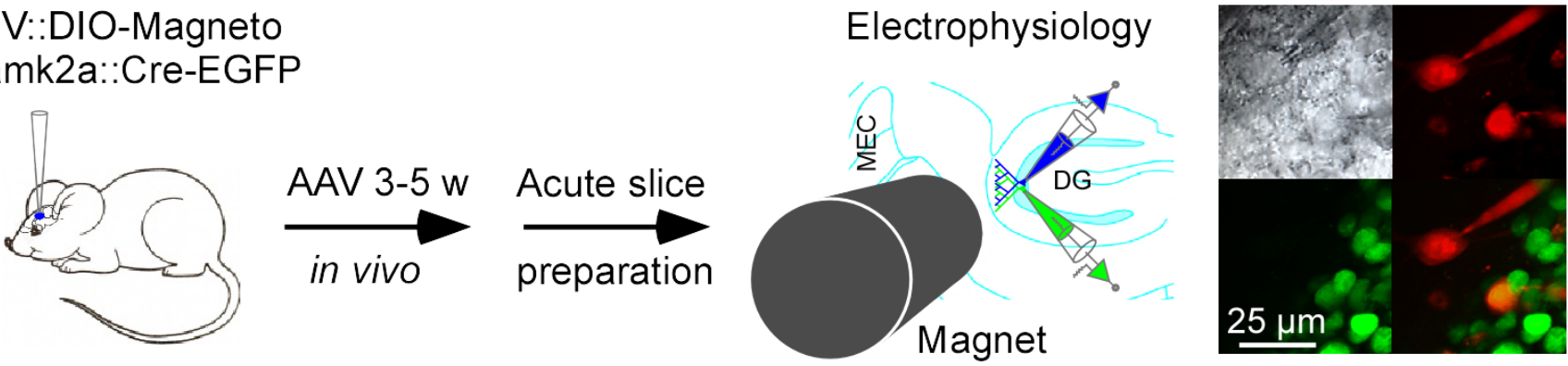

b

- Control - Magneto - Magnet position
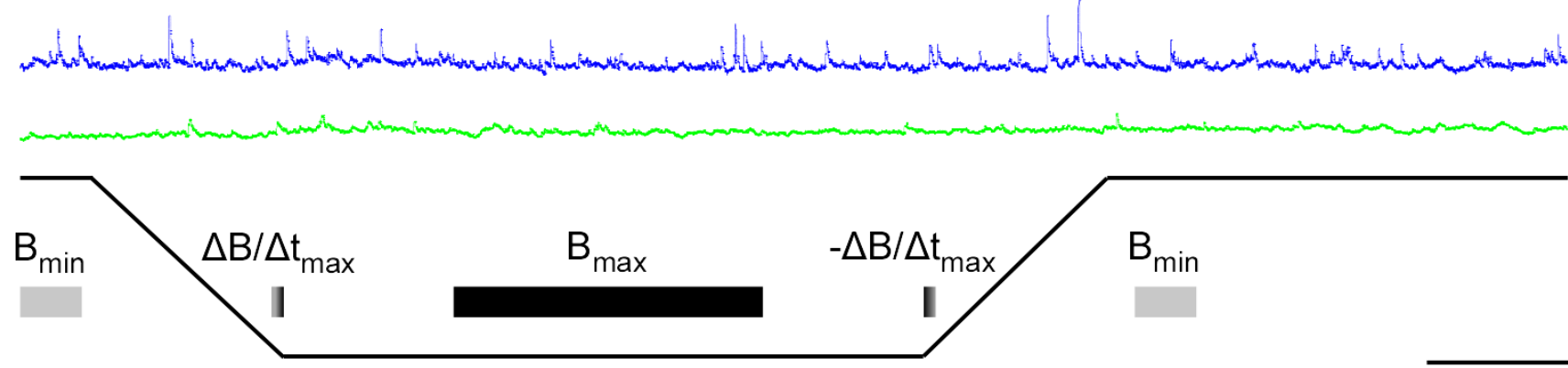

C

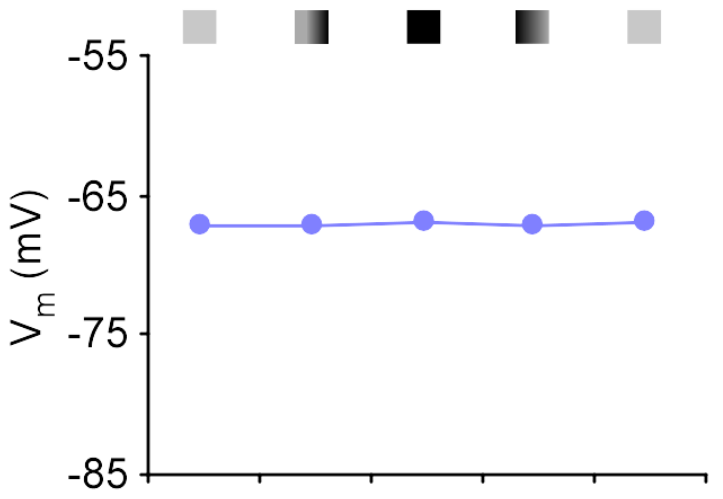

Magneto

d Spontaneous activity in Magneto expressing neuron
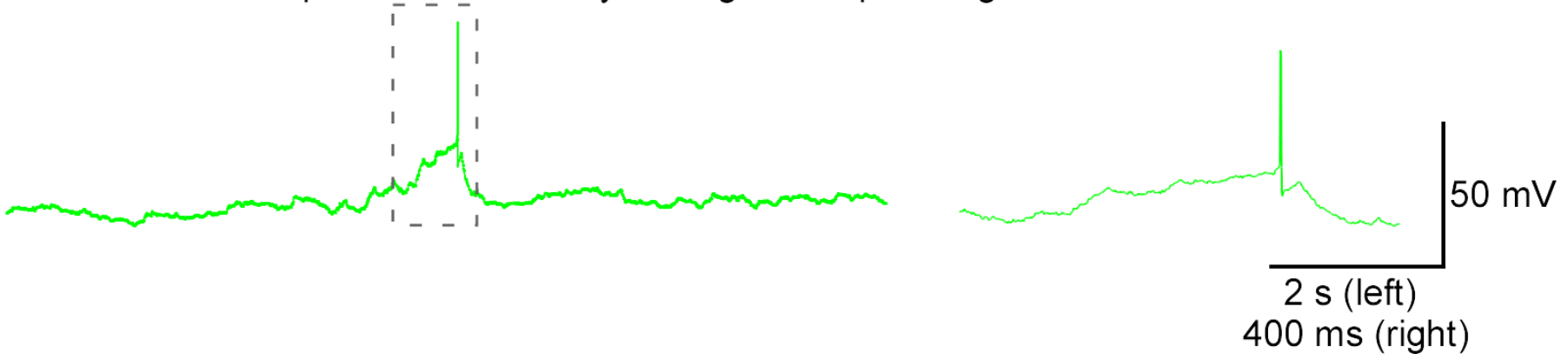

Figure S8. No magnetic effect in mouse hippocampal dentate gyrus neurons expressing Magneto2.0. 
(a) Schematic drawing outlines the design of in vivo AAV viral expression of CMV::DIO-Magneto and Camk2a::Cre-EGFP, ex vivo magnetic stimulation and electrophysiological recordings in acutely prepared mouse hippocampal slices. The right images show simultaneous whole-cell recordings from a pair of control non-expressing and DIO-Magneto/Cre-EGFP expressing hippocampal dentate gyrus (DG) neurons under transmitted light (upper left) and fluorescence microscopy with RFP (upper right) and GFP (lower left) filters and their overlay (lower right). DG: dentate gyrus; MEC: medial entorhinal cortex. Note that the viral expression and acute slice preparation were carried out by Drs Ronald Gaykema and Manoj Patel.

(b) Recordings of membrane potentials of the pair of control non-expressing and DIO-Magneto/Cre-EGFP expressing DG neurons before, during and after magnetic stimuli delivered with a 3/8" permanent block magnet mounted on a micromanipulator.

(c) Values of membrane potentials of control non-expressing (Initial $\mathrm{B}_{\min }$ : $-66.9 \mathrm{mV} ; \Delta \mathrm{B} / \Delta \mathrm{t}_{\max }$ : $-66.8 \mathrm{mV}$; $\mathrm{B}_{\max }$ : 66.8 mV; - $\Delta \mathrm{B} / \Delta \mathrm{t}_{\max }:-66.9 \mathrm{mV}$; Ending $\mathrm{B}_{\min }:-66.8 \mathrm{mV} ; n=1$ ) and DIO-Magneto/Cre-EGFP (Initial $\mathrm{B}_{\min }:-65.6 \pm 2.8$ $\mathrm{mV} ; \Delta \mathrm{B} / \Delta \mathrm{t}_{\max }:-65.7 \pm 2.8 \mathrm{mV}, Z=-1.352, p=0.18 ; \mathrm{B}_{\max }:-65.6 \pm 2.9 \mathrm{mV}, Z=1.181, p=0.24 ;-\Delta \mathrm{B} / \Delta \mathrm{t}_{\max }:-65.6 \pm 2.9 \mathrm{mV}$ $Z=0.854, p=0.40 ;$ Ending $B_{\min }:-65.6 \pm 2.8 \mathrm{mV}, Z=0.854, p=0.40 ; n=7$ ) expressing $\mathrm{DG}$ neurons when the permanent magnet was away from (light), approaching to (light-dark transient color), close to (dark), retracting from (dark-light transient color), and away from (light) recorded neurons. Note dark lines/dotes indicating averages and shaded lines/dotes indicating experimental data. Note also that in this experiment only one control non-expressing neurons was recorded and analyzed with seven DIO-Magneto/GFP-Cre expressing neurons because the majority of dentate gyrus neurons exhibited green fluorescence (see insets).

(d) Recordings of spontaneous events in a DIO-Magneto/Cre-EGFP expressing DG neuron. Note that the spontaneous suprathreshold event in the gray dash line box is shown again in an expanded time scale in the right. 
bioRxiv preprint doi: https://doi.org/10.1101/737254; this version posted August 27,2019 . The copyright holder for this preprint (which was not certified by peer review) is the author/funder, who has granted bioRxiv a license to display the preprint in perpetuity. It is made available under aCC-BY-NC-ND 4.0 International license.

\section{Figure S9}

Magneto-expressing cells
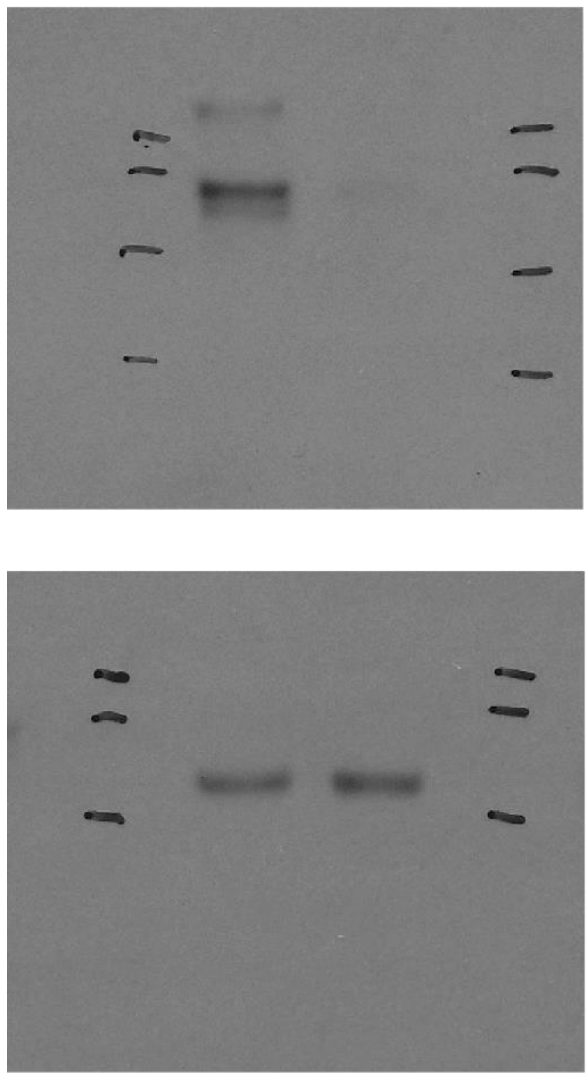

Anti-GluA1

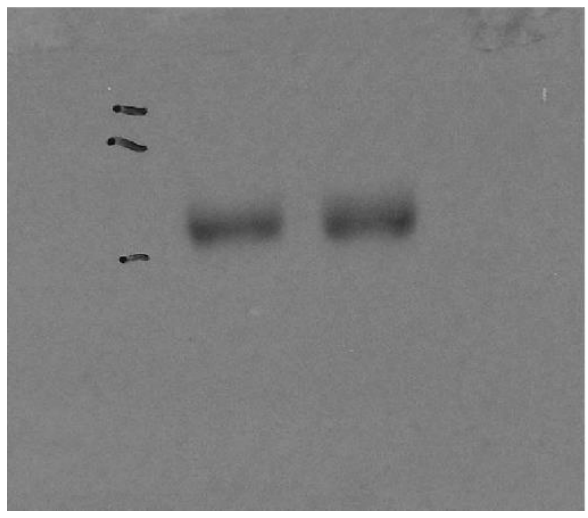

Anti-FLAG
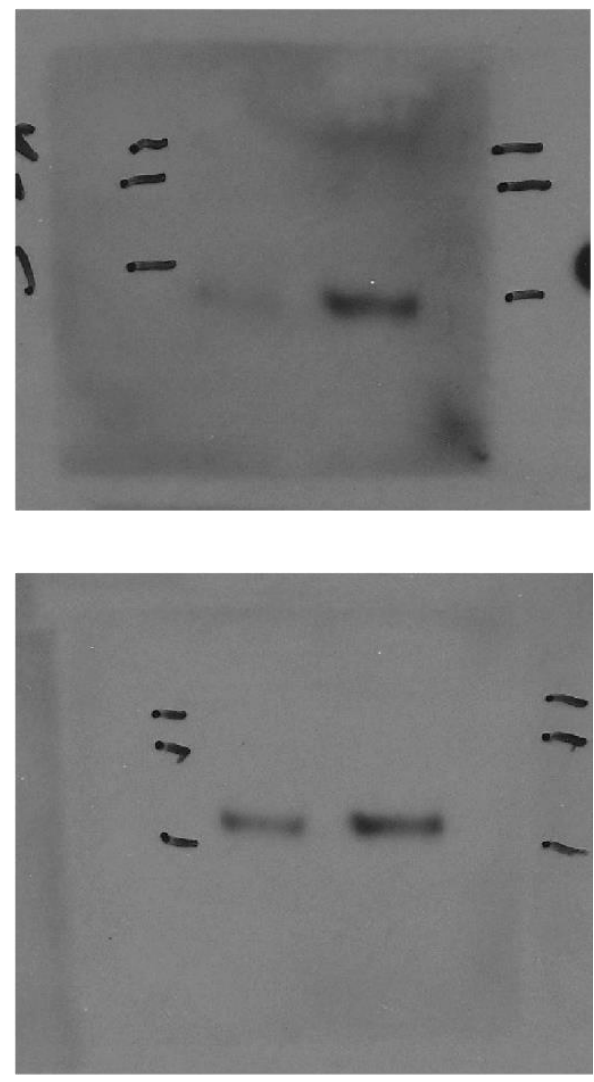

Anti-GluA2

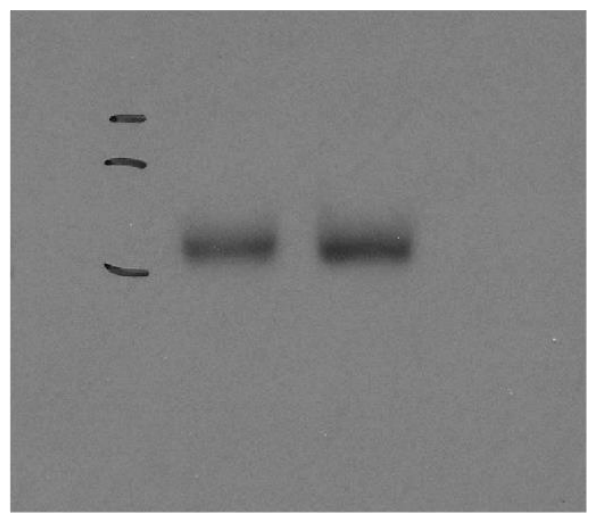

Figure S9. Images of original uncropped Western blots used for preparation of figure 1f-g. 
bioRxiv preprint doi: https://doi.org/10.1101/737254; this version posted August 27,2019 . The copyright holder for this preprint (which was not certified by peer review) is the author/funder, who has granted bioRxiv a license to display the preprint in perpetuity. It is made available under aCC-BY-NC-ND 4.0 International license.

\section{Figure S10}

Magneto-expressing cells
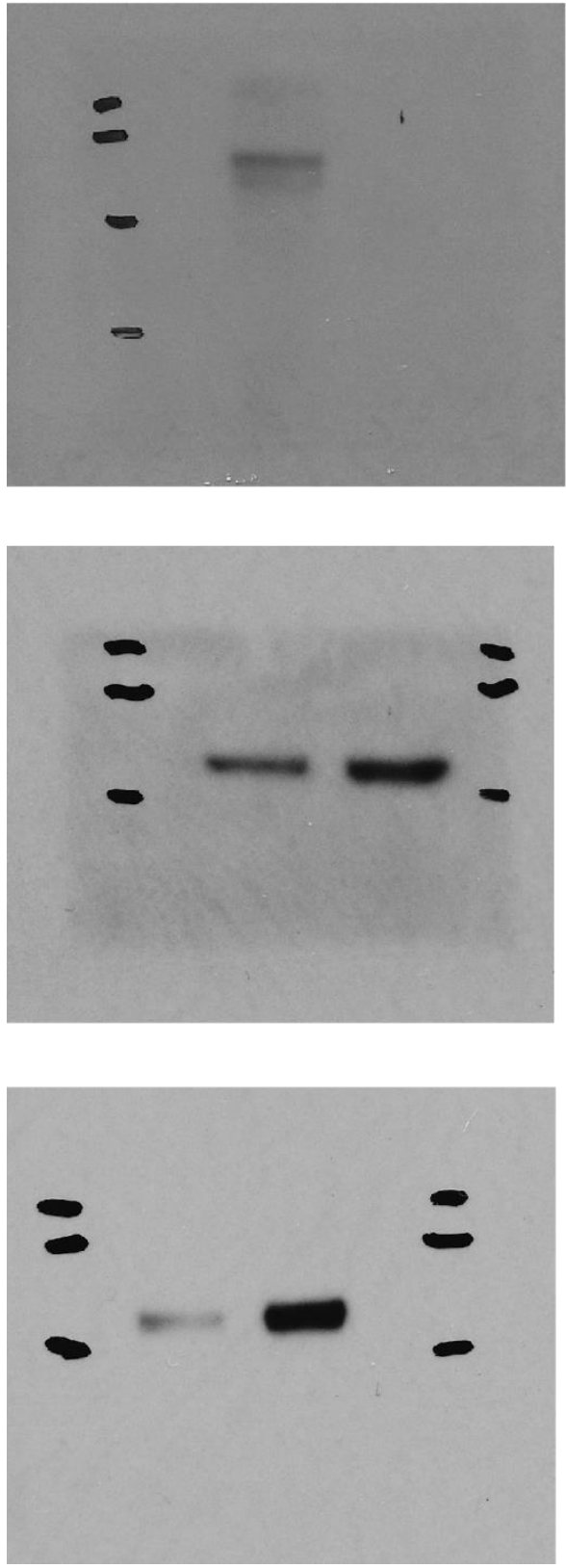

TRPV4-expressing cells

Anti-FLAG

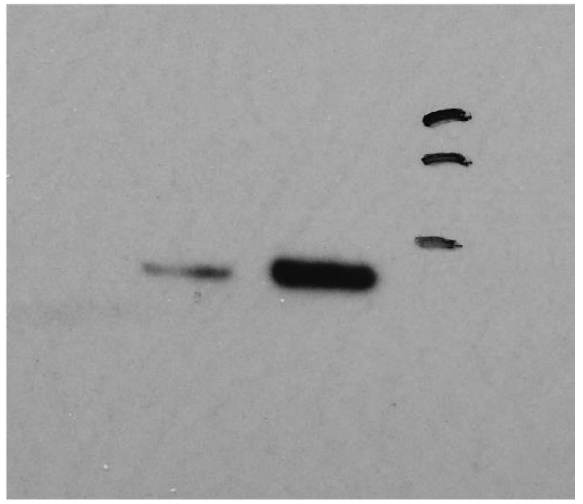

Anti-GluA1

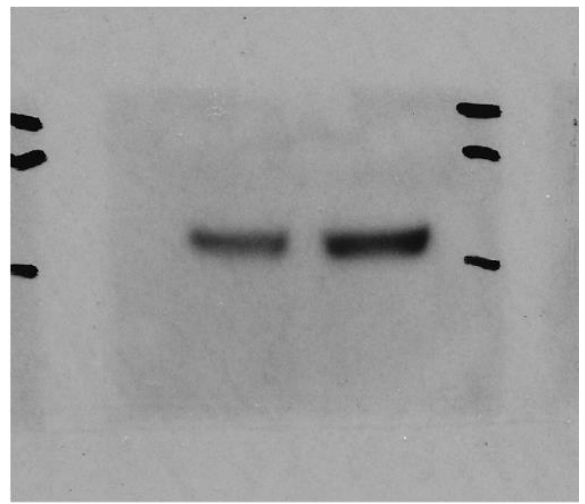

Anti-GluA2

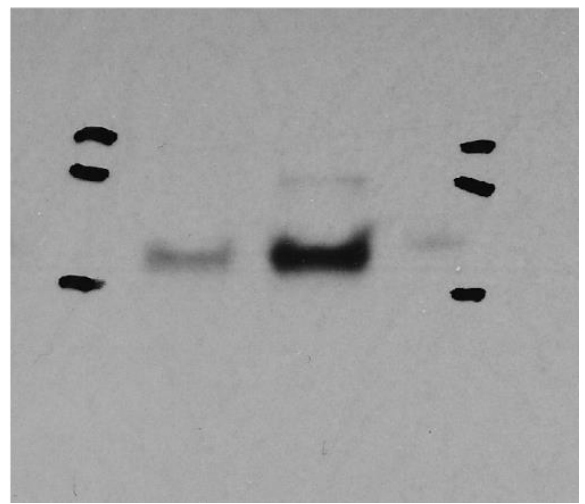

Figure S10. Images of original uncropped Western blots used for preparation of figure S3. 


\section{REFERENCES}

1 Long, X., Ye, J., Zhao, D. \& Zhang, S. J. Magnetogenetics: remote non-invasive magnetic activation of neuronal activity with a magnetoreceptor. Science bulletin 60, 2107-2119, doi:10.1007/s11434-015-0902-0 (2015).

2 Wheeler, M. A. et al. Genetically targeted magnetic control of the nervous system. Nature neuroscience 19, 756-761, doi:10.1038/nn.4265 (2016).

3 Stanley, S. A. et al. Bidirectional electromagnetic control of the hypothalamus regulates feeding and metabolism. Nature 531, 647-650, doi:10.1038/nature17183 (2016).

4 Meister, M. Physical limits to magnetogenetics. eLife 5, doi:10.7554/eLife.17210 (2016).

5 Nilius, B. \& Szallasi, A. Transient receptor potential channels as drug targets: from the science of basic research to the art of medicine. Pharmacological reviews 66, 676-814, doi:10.1124/pr.113.008268 (2014).

6 Wang, G. et al. Cav3.2 calcium channels control NMDA receptor-mediated transmission: a new mechanism for absence epilepsy. Genes \& development 29, 1535-1551, doi:10.1101/gad.260869.115 (2015).

7 Zhang, L. et al. Ras and Rap signal bidirectional synaptic plasticity via distinct subcellular microdomains. Neuron 98, 783-800, doi:10.1016/j.neuron.2018.03.049 (2018).

8 Pang, K. et al. MagR alone is insufficient to confer cellular calcium responses to magnetic stimulation. Frontiers in neural circuits 11, 11, doi:10.3389/fncir.2017.00011 (2017).

9 Kole, K. et al. Assessing the utility of Magmeto to control neuronal excitability in the somatosensory cortex. Nature neuroscience, in press (2019).

$10 \mathrm{Xu}, \mathrm{F} . \mathrm{X}$. et al. Magneto is ineffective in controlling electrical properties of cerebellar Purkinje cells. Nature neuroscience, in press (2019).

11 Duret, G. et al. Magnetic entropy as a proposed gating mechanism for magnetogenetic ion channels. Biophys J 116, 454-468, doi:10.1016/j.bpj.2019.01.003 (2019).

12 Qin, S. et al. A magnetic protein biocompass. Nature materials 15, 217-226, doi:10.1038/nmat4484 (2016).

13 Stanley, S. A., Sauer, J., Kane, R. S., Dordick, J. S. \& Friedman, J. M. Remote regulation of glucose homeostasis in mice using genetically encoded nanoparticles. Nat Med 21, 92-98, doi:10.1038/nm.3730 (2015).

14 Myers, K. R. et al. Arf6-GEF BRAG1 regulates JNK-mediated synaptic removal of GluA1-containing AMPA receptors: a new mechanism for nonsyndromic X-linked mental disorder. J Neurosci 32, 11716-11726, doi:10.1523/JNEUROSCI.1942-12.2012 (2012).

$15 \mathrm{Lim}, \mathrm{C}$. S. et al. BRaf signaling principles unveiled by large-scale human mutation analysis with a rapid lentivirus-based gene replacement method. Genes \& development 31, 537-552, doi:10.1101/gad.294413.116 (2017).

16 Wang, G. et al. An optogenetics- and imaging-assisted simultaneous multiple patch-clamp recordings system for decoding complex neural circuits. Nature protocols 10, 397-412 (2015). 